\title{
Increased Mitochondrial Calcium Uptake and Concomitant Hyperactivity by Presenilin Loss Promotes mTORC1 Signaling to Drive Neurodegeneration
}

\author{
Kerry C. Ryan \\ Albany Medical College \\ Zahra Ashkavand \\ Albany Medical College \\ Shaarika Sarasija \\ Albany Medical College \\ Jocelyn T. Laboy \\ Albany Medical College \\ Rohan Samarakoon \\ Albany Medical College \\ Kenneth Norman ( $\nabla$ normank@mail.amc.edu ) \\ Albany Medical College https://orcid.org/0000-0002-0773-9073
}

Research article

Keywords: Mitochondria, autophagy, presenilin, mTOR, Alzheimer's disease, calcium, Caenorhabditis elegans, neurodegeneration, proteostasis

Posted Date: December 8th, 2020

DOI: https://doi.org/10.21203/rs.3.rs-120594/v1

License: (c) (i) This work is licensed under a Creative Commons Attribution 4.0 International License. Read Full License 


\section{Abstract}

\section{Background}

Metabolic dysfunction and protein aggregation are common characteristics that occur in age-related neurodegenerative disease, such as Alzheimer's disease (AD). However, the mechanisms underlying these abnormalities remain poorly understood. Mutations in the presenilin genes are the primary cause of early onset familial $A D$, but despite their identification over 20 years ago, their role in the disease remains unclear.

\section{Methods}

The model system Caenorhabditis elegans was utilized to study the in vivo function of the highly conserved presenilin ortholog SEL-12 in the nervous system. Cell biological and biochemical assays were employed to monitor changes to proteostasis and autophagic flux in sel-12 mutants. Immunoblotting was used to assess alterations to the activity of the mTORC1 pathway, a central inhibitor of autophagy. Genetic and pharmaceutical strategies to reduce mTORC1 activity, and fluorescent reporters and biosensors were expressed in the mechanosensory neurons to measure mTORC1's influence on proteotoxicity, neuronal health and mitochondrial morphology. Additionally, behavioral response to touch was employed to determine the role mTORC1 activity has in neuronal function in sel-12 mutants. RNA interference by standard feeding methods was used to assess the contribution of autophagy to mTORC1mediated sel-12 defects.

Results

Loss of SEL-12 results in the hyperactivation of the mTORC1 pathway and mTORC1-dependent reduction in autophagy. This hyperactivation is caused by elevated mitochondrial calcium signaling and concomitant mitochondrial hyperactivity. Reducing mTORC1 activity improves proteostasis defects and neurodegenerative phenotypes associated with loss of SEL-12 function. Consistent with high mTORC1 activity, we find that SEL-12 loss reduces autophagy, and this reduction is prevented by limiting mitochondrial calcium uptake or mitochondrial respiration. Moreover, the improvements in proteostasis and neuronal defects in sel-12 mutants due to mTORC1 inhibition require the induction of autophagy.

\section{Conclusion}

SEL-12 has a critical role in mediating mitochondrial calcium homeostasis and activity. In the absence of presenilin function mitochondrial calcium uptake and mitochondrial activity is increased. This mitochondrial hyperactivity stimulates mTORC1 signaling, which inhibits autophagy and promotes proteostasis decline and neuronal dysfunction in sel-12 mutants. These data suggest that the mTORC1 pathway is a potential therapeutic target for treating $A D$.

\section{Background}


The prevalence of neurodegenerative diseases is constantly increasing in the ever-expanding elderly population. These diseases, such as Alzheimer's disease (AD), Huntington's disease, Lewy body dementia, and Parkinson's disease, all share a similar neuropathology that involves protein misfolding and aggregation. For example, $A D$ is associated with protein aggregation in the form of amyloid plaques and neurofibrillary tangles. Biological processes that maintain protein homeostasis (proteostasis) decline with age and this decline is closely linked with neurodegenerative diseases (1-3). However, the mechanisms underlying this decline during aging and neurodegenerative disease progression are not clear.

Mutations in the presenilin encoding genes (PSEN1 and PSEN2) are the primary cause of early-onset, familial AD (FAD), but how presenilin dysfunction promotes neurodegeneration remains disputed. Presenilins are a highly conserved family of proteins that commonly reside on endomembranes such as the endoplasmic reticulum (ER) in organisms as diverse as plants to humans (4). Although presenilin 1 and 2 are primarily known to function as the catalytic component of the $y$-secretase complex, which is involved in the cleavage of the amyloid precursor protein (APP) to produce amyloid beta (A $)$ peptides, many studies have demonstrated a critical $Y$-secretase independent role for presenilins in calcium homeostasis, mitochondrial and lysosome function and autophagy (5-10). However, their function in these cellular processes is far from certain. Importantly, many of these cellular processes are reported to be disrupted in $A D$ and other neurodegenerative diseases (11-13). Therefore, discovering the role of presenilin in these functions may help elucidate the underlying cause of $A D$ and provide insight into how to treat neurodegenerative diseases.

Since presenilin proteins are highly conserved, we have turned to the invertebrate model system Caenorhabditiselegans to help identify the role presenilin may have in the nervous system and understand why presenilin mutations cause neurodegeneration. $C$. elegans provides a novel system for studying presenilin function because it does not produce $A \beta$ peptides $(14,15)$ and, thus, can help resolve the role of presenilin without $A \beta$ accumulation confounding interpretations of presenilin function. Moreover, like the mammalian central nervous system, most $C$. elegans tissues are post-mitotic. Therefore, they cannot use cell division to dilute damage organelles or protein aggregates and, hence, depend on efficient proteostatic pathways to clear faulty proteins and organelles. From our investigations, we have found that mutations in the $C$. elegans presenilin ortholog, SEL-12, similar to mutations in human presenilin, results in altered ER calcium signaling, ER-mitochondrial communication and mitochondrial function $(5,12,16-22)$. In sel-12 mutants, neuronal mitochondrial calcium levels are elevated, which results in mitochondrial hyperactivity and an increase reactive oxygen species (ROS) production. Importantly, reduction of ER calcium release or mitochondrial calcium uptake rescues neuronal as well as mitochondrial function and prevents the neurodegenerative phenotypes observed in sel-12 mutants (23). Additionally, this altered ER-mitochondrial calcium signaling and mitochondrial hyperactivity leads to profound defects in proteostasis and has been found to be independent of gammasecretase activity (16). Furthermore, examining cells from AD patients with PSEN1 mutations, others and we have found that mitochondrial hyperactivity promotes elevated ROS production that can be prevented by blocking mitochondrial calcium uptake $(18,23)$. Thus, a vital question in understanding presenilin 
function concerns the identification of the molecular pathways connecting mitochondrial calcium uptake to neurodegeneration and loss of proteostasis.

Here, we report that the increased mitochondrial calcium uptake and associated mitochondrial hyperactivity accompanying SEL-12 loss drives activation of the mTORC1 (mechanistic target of rapamycin complex I) pathway, which in turn impacts proteostasis and promotes neuronal dysfunction. We show that both genetic and pharmacological inhibition of mTORC1 greatly improves proteostasis in sel-12 mutants. Furthermore, we demonstrate that sel-12 mutants have reduced autophagy that results from mTORC1 signaling activity. Finally, we show that improvements to proteostasis, neuronal function and neuronal health from mTORC1 inhibition in sel-12 mutants require the induction of autophagy. Our data indicate that elevated mitochondrial calcium uptake and mitochondrial hyperactivity resulting from SEL-12 loss disrupts proteostasis through mTORC1 activation and mTORC1-mediated suppression of autophagy, resulting in neurodegeneration.

\section{Methods}

\section{C. elegans maintenance and strains}

For all experiments, C. elegans strains were grown on E. coli OP50 seeded NGM plates at $20^{\circ} \mathrm{C}$. Animals were age synchronized by bleaching gravid worms to obtain the eggs, which were then incubated in $M 9$ for 24-48 hours before being allowed to hatch. Afterward, L1 larvae were grown to adulthood on NGM plates for further experiments. Day 1 adults were analyzed for all experiments unless otherwise indicated. For Q35 aggregation experiments, L4 animals were sterilized by moving them to plates containing 0.5 $\mathrm{mg} / \mathrm{ml}$ 5-fluorouraci1-2'-deoxyribose (Sigma) until the age required for the experiment was reached.

The following strains were used: N2 was the wild type, sel-12(ty11) X, raga-1(ok386) II, uthls248 [aak2p::aak-2(genomic aa1-321)::GFP::unc-54 3'UTR + myo-2p::tdTOMATO], rsks-1(ok1255) III, bzls166 [mec4p::mCherry], zdls5 [mec-4p::GFP + lin-15(+)] I, sqls13 [lgg-1p::GFP::lgg-1 + odr-1p::RFP], dvls100 [unc54p::A-beta-1-42::unc-54 3'-UTR + mtl-2p::GFP], rm/s132 [unc-54p::Q35::YFP], rict-1(ft7) II, mgls77 [rp/28p::ub(G76V)::GFP + unc-119(+) + myo-2p::mCherry] V, js/s609 [mec-4p::MLS::GFP], takEx612 [mec7p::mito-GCaMP6f::SL2:::wrmScarlet], goels22 [mec-4p::SL1::GCaMP3.35::SL2::mKate2:: unc-54 3'UTR + unc-119(+)]. wbmEx238 [rab-3p:::raga-1 cDNA::SL2::mCherry::unc-54 3'UTR]. Genotypes were determined by PCR and DNA sequencing.

\section{Analysis of neuronal morphology}

The structure of the mechanosensory neurons was observed in either mec-7p::GFP(zdls5) or mec$4 p:: m C h e r r y(b z l s 166)$-expressing animals. Presence or absence of wave-like bending in the axon, lesions sprouting off the axon, or a beading-like pattern indicating breaks was determined. The number of sprouts stemming from the ALM soma were counted. The worms were immobilized in $0.3 \%$ sodium azide on $2 \%$ agarose pads, and imaged using the $63 \mathrm{X}$ oil objective on a Zeiss Axio Observer microscope 
equipped with an Andor Clara charged-coupled device (CCD) camera, and Metamorph software was used to compile the images.

\section{Mitochondrial Organization Analysis}

The organization of the mitochondria in the ALM mechanosensory neuronal soma was observed in animals expressing mec-4p::MLS::GFP(js/s609). Animals were immobilized in $1 \mathrm{M}$ levamisole on $2 \%$ agarose pads, then imaged using 100X oil objective on a Zeiss Axio Observer microscope equipped with an Andor Clara CCD camera, and Metamorph software was used to compile the images. Mitochondrial organization was scored on a binary scale, where continuous mitochondria did not show breaks and discontinuous mitochondria appeared fragmented and showed breaks.

\section{Heat stress assay}

For each strain, approximately 50 age-synchronized day 1 adults were placed in a water bath at $37^{\circ} \mathrm{C}$ for $1,2,3$, and 4 hours, then allowed to recover at $20^{\circ} \mathrm{C}$ for 2 hours. Animals were scored as dead if they did not show any movement when prodded with a wire pick.

\section{Mechanosensation assay}

Day 1 adults' response to soft touch was determined using an eyebrow hair attached to a Pasteur pipette, with ten touches per worm, and with each stroke alternating across the anterior and posterior half of the worm as previously described (23). A positive response was scored when the animal moved away from the hair. The mean percentage of positive responses per worm was then determined.

\section{Locomotion assay}

Day 1 adults were placed in a well that was coated with $2 \%$ agarose and filled with 150 uL M9 buffer. A single worm was transferred to an unseeded plate to remove OP50, then picked into the M9 buffer. After waiting one minute for the worm to acclimate, the number of full body bends was counted for one minute.

\section{Quantitation of polyQ aggregates}

unc-54p::Q35::YFP(rm/s132)-expressing day 3 adult animals were immobilized in $0.1 \mu \mathrm{m}$ diameter polystyrene microspheres (Polysciences) on $2 \%$ agarose pads, and were imaged using the $10 \mathrm{X}$ objective on a Zeiss Axio Observer microscope equipped with an Andor Clara CCD camera. Metamorph software was used to compile the images. The number of polyQ aggregates was counted, each aggregate determined as a structure fully discernible from any surrounding aggregates.

\section{Autophagosome assay}

We utilized animals expressing the lgg-1p::/gg-1::GFP (sq/s13) construct to label autophagosomes (24). Animals were immobilized in $0.1 \mu \mathrm{m}$ diameter polystyrene microspheres (Polysciences) on $2 \%$ agarose 
pads. The number of aggregates per visible seam cell or per visible muscle cell was counted. Animals were viewed at 63x magnification using Zeiss AxioObserver microscope equipped/captured with an Andor Clara CCD camera, and Metamorph software was used to compile the images.

\section{Proteosome function assay}

rpl-28p::ub(G76V)::GFP(mg/s77)-expressing day 1 adult animals were immobilized in $1 \mathrm{M}$ levamisole on $2 \%$ agarose pads, and were imaged using the 10X objective on a Zeiss Axio Observer microscope equipped with an Andor Clara charged-coupled device CCD camera. Metamorph software was used to compile the images. The fluorescence intensity was quantified using ImageJ.

\section{Western blot analysis}

Day 1 adult worms were washed with PBS, then lysed via sonication in RIPA buffer with protease (Roche) and phosphatase inhibitors (Roche). For p-RSKS-1 analysis, half the sample was lysed in RIPA buffer with protease inhibitors to determine protein concentration using a BCA assay (Pierce), while the other half was lysed in $2 x$ Laemmli sample (BioRad) buffer containing $5 \%$ beta-mercaptoethanol for use in the assay. 15 ug of each sample was separated with a $10 \%$ tris-glycine gel (BioRad), or with $12 \%$ tris-glycine gel (BioRad) for GFP::LGG-1 samples, then transferred to a $0.2 \mu \mathrm{m}$ nitrocellulose membrane (Invitrogen). The membrane was incubated in TBS with primary antibody overnight (phospho-Drosophila p70 S6 Kinase (Thr398), 1:500, Cell Signaling \#9209; GFP, 1:1000, Cell Signaling \#2555; and beta-actin, 1:1000, MP Biomedicals \#8691002). Fluorescent tagged secondary antibodies were used (IRDye 800CW Goat anti-rabbit (LI-COR), 1:20,000 and IRDye 680RD Goat anti-mouse, 1:20,000 (LI-COR)). The blot was imaged using LiCor Odyssey CLx infrared imaging system and quantified with the Odyssey Image Studio software.

\section{Calcium Imaging}

Mitochondrial calcium was measured in the mechanosensory neurons in animals expressing mec7p::mito-GCaMP6f::SL2:::wrmScarlet as previously described (23). In brief, animals were immobilized on $1 \mathrm{M}$ levamisole on $2 \%$ agarose pads. Images were taken using a $100 \mathrm{X}$ objective lens on a Zeiss Axio Observer microscope equipped with an Andor Clara CCD camera, and images were compiled with Metamorph software. Fluorescence intensity of GCaMP6 was normalized to wrmScarlet fluorescent intensity, which was used as an expression control, and quantified using ImageJ. Cytoplasmic calcium levels were measured similarly in the mechanosensory neurons of animals expressing mec$4 p:: S L 1:: G C a M P 3.35:: S L 2:: m K a t e 2$, with fluorescence intensity of GCaMP6 normalized to mKate2 intensity using ImageJ.

\section{RNAi}

RNAi was delivered by feeding as previously described (25). L1 animals were grown to adulthood on NGM plates seeded with HT115 bacteria expressing lgg-1 or bec-1 double stranded RNA, both from the Ahringer library (26), or empty RNAi feeding vector. RNAi bacteria strains were verified by PCR and DNA 
sequencing. Furthermore, to show specificity $\operatorname{lgg}-1(R N A i)$ and bec-1(RNAi) treatment abolished or reduced lgg-7::GFP, respectively.

\section{Drug treatments}

Rapamycin was prepared in dimethyl sulfoxide (DMSO) and added to plate agar at $100 \mu \mathrm{M}$. Equivalent DMSO was added to control plates. Bortezomib (LC Laboratories) was prepared in DMSO and added to plate agar at $10 \mu \mathrm{M}$. Doxycycline was added as described (23), with doxycycline prepared in and added to plates at a concentration of $10 \mu \mathrm{g} / \mathrm{ml}$. Equivalent DMSO was also used in control plates. For rapamycin, doxycycline, and bortezomib treatments, L4 animals were grown on treated plates overnight, and the assays performed the following day on day 1 adults.

\section{Statistical Analyses}

All statistical analyses were conducted using Graph Pad Prism software. A p value of less than 0.05 is considered to be significant. Statistical difference was determined using a Student's $T$ test for comparing two variables and a one-way analysis of variance with a Tukey post hoc analysis for comparing more than two variables.

\section{Results}

\section{sel-12 mutants show decreased autophagy that is rescued by reducing mitochondrial calcium uptake}

Like other age-related diseases, $A D$ is associated with a general decline in proteostasis that contributes to the buildup of toxic protein aggregates, leading to neuronal dysfunction and death $(27,28)$. It has been postulated that initial impairments to proteostasis pathways promote the accumulation and aggregation of misfolded proteins observed in neurodegenerative disorders, including the accumulation of the aggregation-prone $A \beta$ and tau, whose plaque deposition and tangle formation, respectively, are considered the hallmarks of AD (29-33). Utilizing several models of proteotoxicity, we previously reported that sel-12 mutants have a severe defect in proteostasis resulting from elevated ER to mitochondrial calcium signaling (16). Two major systems are responsible for degrading misfolded or damaged proteins and are critical for maintaining integrity of the proteome: the ubiquitin-proteasome system and the autophagy-lysosomal pathway. To investigate the activity of these pathways in sel-12 mutants, we first examined proteasome activity. As a reporter for proteasome function, we analyzed animals expressing ubiquitin ( $\mathrm{ub}(\mathrm{G} 76 \mathrm{~V})$ ) tagged to GFP, which is readily degraded by the proteasome in wild type animals, but accumulates if proteasome activity is perturbed (34) (Fig. 1A). Indeed, unlike animals treated with a proteasome inhibitor (bortezomib), animals carrying a sel-12 null allele, sel-12(ty 11), showed similarly low fluorescent intensity as wild type animals (Fig. 1A-B), thus, indicating typical proteasomal degradation of the ub(G76V) tagged GFP. These data are consistent with normal proteasome activity of sel-12 mutants previously reported (16). 
Next to investigate the activity of autophagy-lysosomal pathway in sel-12 mutants, we utilized GFP tagged LGG-1 to visualize autophagosome formation. LGG-1 is the $C$. elegans ortholog of LC3/Atg8, which is incorporated into and decorates pre-autophagosomal and autophagosomal membranes and organizes into puncta during autophagy and, thus, is a widely used autophagy marker (35-37). Compared to wild type animals, we observed a significant reduction in the number of LGG-1::GFP puncta in the hypodermal seam cells (Fig. 1C-D) and body wall muscle (Fig. 1E-F) of sel-12 mutants. To determine whether elevated ER-mitochondrial calcium signaling in sel-12 mutants is responsible for decreased puncta formation, we introduced into the sel-12 mutant background a null mutation in the mitochondrial calcium uniporter, $m c u-1$, which reduces mitochondrial calcium uptake $(23,38)$ and has been shown to reduce mitochondrial calcium levels, neurodegenerative phenotypes and proteostasis defects in sel-12 mutants $(16,23)$. We found that introduction of an mcu- 1 null mutation in the sel-12 mutant background increases puncta formation to levels indistinguishable from wild type animals (Fig. 1G). This suggests that altered mitochondrial calcium signaling is responsible for reducing autophagy in sel-12 mutants. As an alternative method to measure autophagic flux, we immunoblotted for GFP in the GFP::LGG-1 animals to assess lipidation of LGG-1 with phosphatidylethanolamine (PE). When autophagy is induced, $P E$ is conjugated to LGG-1 to anchor it to the autophagosome. Thus, LGG-1-PE detection via western blotting is a reliable method to quantify the rate of autophagy (39). Consistent with this, GFP::LGG-1-PE levels significantly increase in raga-1 null mutants, which have been shown to have elevated autophagy via inhibition of mTORC1 signaling (40) (Fig. S1A,B). Consistent with our autophagosome puncta quantification, we found the amount of processed GFP::LGG-1-PE was significantly decreased in sel-12 mutants, and the level of processed GFP::LGG-1-PE was restored in mcu-1; sel-12 animals (Fig. 1H,I). Therefore, unlike proteasome activity in sel-12 mutants, these data suggest that autophagy is defective, which is consistent with previous observations of disrupted autophagy in sel-12 mutants as well as other models studying presenilin function $(6,9,16,41,42)$ and also implicate a critical role of mitochondrial calcium in this defect.

\section{Inhibition of mTORC1 in sel-12 mutants increases autophagy}

A central inhibitor of autophagy is the serine/threonine protein kinase mTORC1 signaling pathway (43, 44). To investigate whether mTORC1 has a role in inhibiting autophagy in sel-12 mutants, we genetically ablated two key positive mediators of the mTORC1 pathway in sel-12 mutant animals. These include the gene encoding the RagA GTPase ortholog, raga-1, which is critical for the activation of MTORC1; and a gene encoding a key effector protein of mTORC1 signaling, ribosomal protein S6 kinase (rsks-1). Analysis of LGG-1::GFP puncta in raga-1(ok386); sel-12 and rsks-1(ok1255); sel-12 double mutant animals reveals that sel-12 mutants with mTORC1 signaling inhibited, unlike sel-12 mutants alone, show robust accumulation of LGG-1::GFP puncta (Fig. 1C-F). Moreover, consistent with mTORC1 acting as a strong inhibitor of autophagy, blocking mTORC1 signaling resulted in elevated puncta formation and GFP::LGG1-PE levels in wild type animals (Fig. 1C-F, Fig. S1A,B). Notably, a similar number of puncta is observed in sel-12 mutants with compromised mTORC1 signaling (Fig. 1C-F). These data indicate that sel-12 mutants have the capacity to carry out autophagy when mTORC1 signaling is disrupted. However, without 
mTORC1 inhibited, autophagy is blunted in sel-12 mutants, suggesting a role of activated mTORC1 in mediating sel-12 phenotypes.

\section{mTORC1 signaling is upregulated in sel-12 mutants.}

Given that the mTORC1 pathway is a central inhibitor of autophagy and is a critical metabolic sensor (43, 44) and we have observed increased mitochondrial metabolic activity due to elevated ER to mitochondria calcium signaling in sel-12 mutants (23), we asked whether mTORC1 signaling is elevated in sel-12 mutants. To assess mTORC1 activity, we examined phosphorylation levels of the central mTORC1 target RSKS-1/S6 kinase in sel-12 mutants. Strikingly, phosphorylated RSKS-1 was significantly increased in sel12 animals compared to wild-type animals (Fig. 2A,B), indicating that mTORC1 signaling is elevated in sel-12 mutants. Next, we sought to determine whether the increased mitochondrial activity observed in sel-12 mutants is leading to the elevation in MTORC1 signaling. Previously, we demonstrated that loss of sel-12 function promotes calcium uptake into the mitochondria from the ER, a process that increases mitochondrial activity and leads to the subsequent proteostatic collapse and neurodegeneration observed in sel-12 mutants $(16,23)$. Indeed, reducing ER calcium release or mitochondrial calcium uptake in sel-12 mutants, as well as reducing oxidative phosphorylation, suppresses the proteostasis defects and neurodegeneration phenotypes observed in sel-12 mutants $(16,23)$. To determine whether this altered calcium signaling pathway observed in sel-12 mutants is responsible for mTORC1 hyperactivation, we examined phospho-RSKS-1 levels in mcu-1; sel-12 double mutants and found that introduction of the mcu-1 null mutation leads to reduced phopsho-RSKS-1 levels in sel-12 mutants compared to sel-12 mutants alone (Fig. 2C,D). It is likely that mitochondrial calcium levels are responsible for changes to mTORC1 activation, as the mcu-1 mutation does not increase cytosolic calcium levels relative to the sel12 mutation alone (Fig. S2). To determine whether mitochondrial hyperactivity due to elevated mitochondrial calcium promotes mTORC1 activation, we treated sel-12 worms with doxycycline to reduce mitochondrial respiration $(23,45)$. Similar to blocking mitochondrial calcium uptake, sel-12 mutants treated with doxycycline abrogated the increase in phospho-RSKS-1 levels (Fig. 2E,F). Collectively, these data suggest that the altered ER-mitochondrial calcium signaling in sel-12 mutants causes aberrant activation of the mTORC1 pathway by increasing mitochondrial activity.

\section{Reduction of mTORC1 signaling suppresses neuronal defects in sel-12 mutants.}

We next asked whether mTORC1 activity contributes to the behavioral and neuronal defects seen with sel12 loss. We first examined the structure of the $C$. elegans mechanosensory neurons, which show age dependent structural decline and neurodegeneration (46-48). Previously, we found that these structural aberrations associated with aging develop precociously in sel-12 mutants (23). Day 1 adult sel-12 mutants display numerous ectopic neurite sprouts stemming off the ALM neuronal soma which are absent in wild type animals, and also show defects in the structure of the ALM and PLM axons, exhibiting abnormal lesions at a higher frequency relative to wild-type animals at day 1 (Fig. 3A-C, Fig. S3A,C). To determine whether mTORC1 inhibition can suppress these neuronal morphological defects, we examined mechanosensory neuron structure in rsks-1; sel-12 and raga-1; sel-12 animals, as well as in aak- 
2(uth/s248); sel-12 animals, which carry a mutation that results in constitutive activation of the catalytic subunit of $5^{\prime}$ adenosine monophosphate-activated protein kinase (AMPK/AAK-2), a global energy sensor and a major inhibitor of mTORC1 activity (49). raga-1; sel-12, aak-2(uth/s248); sel-12, and rsks-1; sel-12 animals each showed substantially improved neuronal morphology, with reduced ectopic neurite processes stemming off the soma (Fig. 3B) and reduced frequency of lesions (Fig. 3C), wave-like processes (Fig. S3B) and breaks (Fig. S3C) in the ALM and PLM axons. In addition, we treated the sel-12 animals with rapamycin, a clinical grade drug that specifically inhibits mTORC1, and examined neuronal morphology. Consistent with the genetic manipulations, treatment with rapamycin showed similar improvements to the ALM soma (Fig. 3D) and axonal structure (Fig. 3E, Fig. S3D,E).

To determine whether the structural improvements we observed in the mechanosensory neurons translates to functional improvement, we examined the effects of genetic and pharmacological mTORC1 inhibition on soft touch behavior, which is controlled by the mechanosensory neurons. Wild type day 1 adult animals, when touched on the anterior or posterior half of the body with an eyebrow hair, will reverse their progression and move away from the stimulus. Consistent with increased morphological defects in the mechanosensory neurons of aged animals, mechanosensation declines with age (47). This reduced response rate happens prematurely in sel-12 mutants and continues to worsen with age (23). Indeed, day 1 adult sel-12 mutants show pronounced defects in soft touch response (Fig. 3F). Consistent with mechanosensory neuronal structural improvements, the raga-1; sel-12, aak-2(uth/s248); sel-12, and rsks-1; sel-12 double mutants all showed significant improvements to soft touch response (Fig. 3F). Moreover, rapamycin treatment recapitulated these improvements (Fig. 3G). Furthermore, this improvement is specific to mTORC1, as inhibition of mTORC2 signaling in sel-12 mutants showed no improvement to sel-12 mutant neuronal defects. Indeed, genetic ablation of RICTOR, rict-1, which is required for the activation of $\mathrm{mTORC2}$, in the sel-12 mutant background did not show significant improvements to mechanosensory neuron morphology or soft touch behavior (Fig. S4). These data indicate that neurodegeneration in sel-12 mutants can be suppressed with mTORC1 inhibition.

Additionally, we found that expression of raga-1 cDNA driven under the pan-neuronal rab-3 promoter (50) was sufficient to fully prevent any improvements to soft touch behavior in raga-1; sel-12 animals (Fig. $3 \mathrm{H}$ ), indicating a cell autonomous role of mTORC1 hyperactivity in the nervous system of sel-12 mutants. Additionally, unlike in wild type animals, pan-neuronal expression of raga-1 in the neurons of sel-12 animals significantly aggravated their touch defect. These data demonstrate a central role for neuronal mTORC1 activity in mediating and exacerbating the neurodegenerative behavioral defect in sel-12 mutants.

\section{Inhibition of mTORC1 improves proteostasis in sel-12 mutants.}

mTORC1 activity may further explain impaired proteostasis in sel-12 animals. In fact, many studies show that modulation of mTORC1 activity widely impacts proteostasis (51). To define a role of mTORC1 in the collapse of proteostasis in sel-12 mutants (16), we first examined animals with body wall expression of polyglutamine (polyQ) Q35::YFP fusion protein (rm/s132), which aggregates as the animals age (52). 
While expression of Q35::YFP remains soluble and evenly distributed in day 3 adult wild type animals, adult sel-12 mutants show premature Q35 aggregation by day 3. However, analyses of raga-1; sel-12 and rsks-1; sel-12 animals show a significant reduction in polyQ aggregates at day 3 compared to sel-12 mutants, suggesting that mTORC1 inhibition improves proteostasis in these animals (Fig. 4A,B). Consistent with these results, rapamycin treatment also reduced polyQ aggregates in day 3 adult sel-12 adult animals (Fig. 4C).

As an alternate method to assess the state of proteostasis in sel-12 mutants, we examined animals expressing human A $1-42$ (dv/s100), which generates proteostatic stress and causes progressive paralysis in the transgenic animals (15). Previously, we found that that sel-12 mutants expressing A 1 1-42 have severely reduced motility relative to either mutant background alone, suggesting that the sel-12 mutation promotes Abeta1-42 toxicity and enhances proteostasis defects (16). To determine the effect of mTORC1 inhibition on motility in this background, we examined swimming behavior of raga-1; sel-12 and rsks-1; sel-12 mutants expressing A $1-42$, and found they had significantly higher motility compared to sel-12 mutants expressing A 1 1-42 (Fig. 4D). Furthermore, treating sel-12 mutants expressing A $1-42$ with rapamycin showed similar improvements in motility (Fig. 4E).

To evaluate the state of proteostasis of endogenous proteins, we subjected animals to heat stress (exposure to $37^{\circ} \mathrm{C}$ ) to induce protein misfolding (53) and then examined animal survival. Previously, we found that sel-12 mutants have reduced resistance to heat stress and reduced survival (16). Thus, we examined the survival rate of wild type, sel-12 and raga-1; sel-12 mutants, as well as rapamycin treated sel-12 animals after $1,2,3$, and 4 hours of exposure to $37^{\circ} \mathrm{C}$. We found that the survival rate after heat stress at each time point was increased in the sel-12 mutants with mTORC1 signaling inhibited either genetically or pharmacologically relative to sel-12 mutants (Fig. 4F,G). Altogether, these data suggest that mTORC1 impacts proteostasis in sel-12 mutants and that the defects in proteostasis due to loss of SEL12 are improved through $\mathrm{mTORC} 1$ inhibition.

\section{Improvements to proteostasis and neuronal function through mTORC1 inhibition require the induction of autophagy.}

To further investigate the mechanism by which MTORC1 contributes to proteostasis and neuronal defects in sel-12 mutants, we first examined the condition of mitochondrial morphology in sel-12 mutants with mTORC1 signaling abrogated. Previously, we found that sel-12 mutants have severe defects in mitochondrial morphology and function due to elevated mitochondrial calcium $(23,54)$. To determine whether mTORC1 activity contributes to mitochondrial disorganization in sel-12 mutants, we examined sel-12 and raga-1; sel-12 mutants expressing a mitochondrial localization signal tagged with GFP in the mechanosensory neurons $(23,55,56)$. We found that compared to sel-12 mutants, raga-1; sel-12 double mutants had significantly improved mitochondrial organization, suggesting that elevated mTORC1 activity contributes to aberrant mitochondrial structure in sel-12 mutants (Fig. 5A,B).

Next, since we previously found that disorganized mitochondrial structure in sel-12 mutants is caused by elevated ER to mitochondrial calcium signaling $(23,54)$, we asked whether the improvements we 
observed in raga-1; sel-12 mutants are due to reduced ER-mitochondrial calcium signaling. Using a genetically encoded GCaMP6 calcium indicator localized to the mitochondrial matrix (23), we assessed mitochondrial calcium levels in the mechanosensory neurons. Notably, we found that calcium levels were unchanged after mTORC1 inhibition (Fig. 5C,D), indicating that mTORC1 activation is likely a downstream consequence of the altered calcium signaling observed in sel-12 mutants. This data is consistent with our pRSKS-1 western blot data showing that reduction of mitochondrial calcium uptake reduced pRSKS-1 levels in the sel-12 mutant background (Fig. 2C,D).

Thus, since mTORC1 is likely not playing a role in mitochondrial calcium signaling in the sel-12 mutants, it is possible mTORC1's inhibition of autophagy is responsible for the defects in proteostasis and neuronal function observed in sel-12 mutants. To determine whether the improvements in behavior and proteostasis through mTORC1 inhibition is primarily due to promoting autophagy, we knocked down inducers of autophagy $l g g-1$ and bec-1 using RNA interference (RNAi). Importantly, RNAi directed to $/ g g-1$ or bec-1, which encodes the $C$. elegans beclin 1 ortholog, have been shown to inhibit autophagy $(37,57)$. From these analyses, we found that the improvements to soft touch behavior in raga-1; sel-12 mutants is abrogated when treated with lgg-1 or bec-1 RNAi (Fig. 6A), and these animals resemble sel-12 mutant animals. Similarly, the improvements to proteostasis as measured by the number of Q35 aggregates in raga-1; sel-12 double mutants are lost with either Igg-1 or bec-1 RNAi treatment (Fig. 6B). Additionally, the increase in swimming rate is lost in raga-1; sel-12 animals expressing A $31-42$ when treated with Igg-1 or bec-1 RNAi (Fig. 6C). These data suggest mTORC1 primarily impacts proteostasis and neuronal function in sel-12 animals by inhibiting autophagy. Altogether, our data identify activation of mTORC1 as a critical pathway by which SEL-12 loss results in neurodegeneration, and define an important role of the mTORC1 pathway in exacerbating the defects in proteostasis and autophagy following loss of SEL-12.

\section{Discussion}

$A D$ is characterized by the pathophysiological buildup of protein aggregates such as amyloid plaques and neurofibrillary tangles. The decline in the ubiquitin-proteasome and the autophagy-lysosomal proteostasis pathways that are responsible for degrading misfolded or damaged proteins has been postulated to underlie age related diseases such as AD, Huntington's disease, Lewy body dementia and Parkinson's disease (1-3). Here, we have shown that the premature decline in proteostasis in animals lacking SEL-12/presenilin function is due, at least in part, to decreased autophagy. Our findings demonstrate that in $C$. elegans the loss of SEL-12/presenilin function leads to elevated mTORC1 signaling and this increase is due to the disruption of mitochondrial calcium homeostasis and mitochondrial hyperactivity. Furthermore, this increase in mTORC1 signaling inhibits autophagy ultimately resulting in the collapse of proteostasis and progression of neuronal dysfunction. Thus, our data indicate a crucial function of SEL-12/presenilin in mediating ER-mitochondrial calcium homeostasis that is vital for proteostasis and disruption of this role leads to neurodegeneration.

Presenilins are a family of highly conserved proteins that are commonly found on endomembranes, such as the ER and endosomes (4). The functional role of the presenilin family on these endomembrane 
structures is not well understood. The best studied function of presenilin is its role as the catalytic subunit of the gamma secretase, an intramembrane aspartyl protease. In addition to presenilin, the gamma secretase is composed of three other well-conserved components, including APH-1, APH2/Nicastrin and PEN-2 $(58,59)$. Gamma secretase cleaves several type I transmembrane proteins most notably Notch and APP (60). It is presenilin's role in the proteolytic cleavage of APP that has drawn the most intensive research efforts. This is because mutations in PSEN1 or PSEN2 or mutations or duplications in APP cause early onset familial AD. Due to the accumulation of $A \beta$ peptides in patients with $A D$ and the ability of the gamma-secretase to process APP to generate $A \beta$ peptides, research on presenilin function has primarily focused on APP processing and the amyloid hypothesis, which centers on $A \beta$ peptide accumulation causing AD. Despite intensive work on the amyloid hypothesis and a deep understanding into the processing of APP, the cause of $A D$ is still not clear and a successful treatment for $A D$ is not available. While smaller than the body of literature covering APP processing, there are many reports of gamma-secretase independent functions of presenilin in regulating several biological pathways that are disrupted in $A D$, such as calcium homeostasis, mitochondrial and lysosomal function and autophagy $(5-9,61)$.

Presenilins found on the ER are enriched in membrane fractions that are associated with mitochondrial contact, the mitochondrial associated membranes (62). Accordingly, disruption of presenilin function results in more frequent ER mitochondrial contacts and signaling shared between these organelles, which leads to altered lipid synthesis and calcium uptake into the mitochondria $(5,12,17,20-22)$. This altered calcium uptake results in mitochondrial functional changes and elevated oxidative stress $(18,23,54)$. In C. elegans, this oxidative stress leads to neuronal dysfunction, which can be suppressed by limiting ER calcium release, mitochondrial calcium uptake or mitochondrial respiration (23). Importantly, this ERmitochondrial activity of presenilin has been found to be gamma-secretase independent $(16,23,61)$. Moreover, in $C$. elegans, we have found that this altered ER-mitochondrial calcium signaling promotes the collapse of proteostasis in sel-12 mutants, which can be reversed by reducing ER calcium release or mitochondrial calcium uptake (16).

Here, we find that the proteostasis defects associated with loss of SEL-12/presenilin are due to a reduction of autophagy caused by elevated mTORC1 activity. Both in vivo models of AD and post-mortem brain samples from AD patients have revealed hyperactivated mTORC1 signaling (63-66). mTORC1 activity promotes cellular growth and metabolism by activating many anabolic pathways, such as the biosynthesis of protein and lipids, and inhibiting catabolic processes, such as autophagy. Despite reports indicating $\mathrm{mTORC} 1$ hyperactivation in $A D$, the cause of $\mathrm{mTORC} 1$ activation is not known. Several studies have suggested $A \beta$ peptides directly or indirectly activate $\operatorname{mTORC} 1(64,67)$ and other studies have suggested that the activation of mTORC1 is an enhancer of $A \beta$ generation and deposition $(68,69)$. Interestingly, several laboratories have shown that hyperactive mTORC1 contributes to tau pathology (e.g. neurofibrillary tangles), which in addition to amyloid plaques, is a classic hallmark of $\operatorname{AD}(68,70,71)$. In C. elegans sel-12 mutants, we find that altered ER-mitochondrial calcium signaling and concomitant mitochondrial hyperactivity increases mTORC1 signaling, which subsequently leads to decreased autophagy. This in turn promotes proteostatic stress in sel-12 mutants driving neuronal dysfunction. 
Since $C$. elegans does not generate A $\beta$ peptides, the mechanism leading to mTORC1 activation in driving neurodegeneration in $C$. elegans is not mediated through $A \beta$ peptides but through altered mitochondrial calcium homeostasis and mitochondrial hyperactivity. Indeed, we demonstrate that reduction of mitochondrial calcium or mitochondrial activity in sel-12 mutants reduces mTORC1 signaling. Importantly, we have shown that reduction of ER calcium release or mitochondrial calcium uptake rescues the proteostasis defects and neurodegeneration observed in sel-12 mutants $(16,23)$. Calcium uptake into the mitochondrial matrix is critical for calcium buffering and influencing mitochondrial metabolic activity (e.g. generation of NADH, ATP and superoxide). Alterations in mitochondrial calcium uptake can lead to oxidative stress or calcium overload triggering cell death (72-74). Interestingly, several recent studies have revealed a role of increased mitochondrial calcium levels in promoting neurodegenerative phenotypes (75-79). For example, in Drosophila and zebrafish, it was found that mutations in the genes encoding the PINK1 ortholog results in elevated mitochondrial calcium, and when mitochondrial calcium was reduced in these mutants neurodegeneration was prevented $(80,81)$. Moreover, it was recently demonstrated in a mouse model of AD that neuronal mitochondrial have increased calcium levels that precedes neurodegeneration and that inhibition of mitochondrial calcium influx prevents neurodegeneration (82).

Our data show that loss of SEL-12/presenilin function causes proteostatic collapse and neurodegeneration, at least in part, by the hyperactivation of $\mathrm{mTORC} 1$ and the ensuing inhibition of autophagy in $C$. elegans. Of note, hyperactivation of mTORC1 signaling is associated with several neurological disorders, such as autism, AD, and tuberous sclerosis, and suppression of mTORC1 using compounds that inhibit mTOR signaling has been shown to be an effective treatment in several clinical trials (83-85). Using $C$. elegans, we have found that treatment with rapamycin, a classic mTORC1 inhibitor, improves the proteostasis and neurodegeneration defects associated with sel-12 mutants. Additionally, several studies have demonstrated the effectiveness of rapamycin on ameliorating neuropathology in mouse AD models overexpressing amyloid beta or hyperphosphorylated Tau (86-89). Thus, these data suggest that treatment with MTOR inhibitors may provide some therapeutic benefit to patients suffering from AD. In summary, our study shows that loss of SEL-12/presenilin results in mTORC1 activation, caused by exacerbated mitochondrial calcium uptake and concomitant mitochondrial hyperactivity, which further contributes to loss of proteostasis and neurodegeneration in sel-12 mutants.

\section{Conclusions}

Collectively, our data reveal a novel connection between dysregulated calcium signaling and mTORC1 hyperactivation in presenilin/ sel-12 mutants. Increased mitochondrial calcium uptake and concomitant mitochondrial hyperactivity accompanying presenilin/SEL-12 loss drives mTORC1 pathway activation, which subsequently leads to a decrease in autophagy. This, in turn, promotes proteostatic stress and neuronal dysfunction. Since $C$. elegans does not generate $A \beta$ peptides, the mechanism leading to mTORC1 activation in driving neurodegeneration in $C$. elegans is not mediated through $A \beta$ peptides but through altered mitochondrial calcium homeostasis and mitochondrial hyperactivity. Indeed, we 
demonstrate that reduction of mitochondrial calcium or mitochondrial activity in sel-12 mutants reduces mTORC1 signaling to wild type levels. Genetic and pharmacological inhibition of mTORC1 also greatly improves proteostasis and neuronal function in sel-12 mutants. Our data identify a crucial function of SEL-12/presenilin in mediating ER-mitochondrial calcium homeostasis that is vital for proteostasis and neuronal health.

\section{Abbreviations}

AD: Alzheimer's disease; APP: amyloid precursor protein; A $\beta$ : amyloid beta; CCD: charged-coupled device; DMSO: dimethyl sulfoxide; ER: endoplasmic reticulum; mTORC1: mechanistic Target of Rapamycin Complex 1; MTS: mitochondrial localization signal; NGM: nematode growth media; PE: phosphatidylethanolamine; PolyQ: polyglutamine; ROS: reactive oxygen species

\section{Declarations}

\section{Acknowledgements}

We thank current and former members of the KRN laboratory for discussions and help; and Drs. M. Driscoll, M. Jansen, W. Mair and N. Morsci as well as the CGC, which is funded by the NIH Office of Research Infrastructure Programs (P40 OD010440), for reagents and nematode strains.

\section{Funding}

This work was supported by NIH grants, GM088213 and AG064175.

\section{Authors' Contributions}

Conceived and designed the experiments: KCR, AZ, SS, KRN; Performed the experiments: KCR, AZ, SS, JTL, RS; Analyzed the data: KCR, KRN; Manuscript preparation: KCR, KRN. All authors read and approved the final manuscript.

\section{Availability of data and materials}

The datasets used and/or analyzed during the current study are available from the corresponding author on reasonable request.

\section{Competing Interests}

The authors declare that they have no competing interests.

\section{Consent for publication}

Not applicable. 


\section{Ethics approval and consent to participate}

Not applicable.

\section{References}

1. Kaushik S, Cuervo AM. Proteostasis and aging. Nat Med. 2015;21(12):1406-15.

2. Labbadia J, Morimoto RI. The biology of proteostasis in aging and disease. Annu Rev Biochem. 2015;84:435-64.

3. Yerbury JJ, Ooi L, Dillin A, Saunders DN, Hatters DM, Beart PM, et al. Walking the tightrope: proteostasis and neurodegenerative disease. J Neurochem. 2016;137(4):489-505.

4. Smolarkiewicz M, Skrzypczak T, Wojtaszek P. The very many faces of presenilins and the gammasecretase complex. Protoplasma. 2013;250(5):997-1011.

5. Cheung KH, Shineman D, Muller M, Cardenas C, Mei L, Yang J, et al. Mechanism of Ca2+ disruption in Alzheimer's disease by presenilin regulation of InsP3 receptor channel gating. Neuron. 2008;58(6):871-83.

6. Lee JH, Yu WH, Kumar A, Lee S, Mohan PS, Peterhoff CM, et al. Lysosomal proteolysis and autophagy require presenilin 1 and are disrupted by Alzheimer-related PS1 mutations. Cell. 2010;141(7):1146-58.

7. Neely KM, Green KN, LaFerla FM. Presenilin is necessary for efficient proteolysis through the autophagy-lysosome system in a gamma-secretase-independent manner. J Neurosci. 2011;31(8):2781-91.

8. Nelson O, Tu H, Lei T, Bentahir M, de Strooper B, Bezprozvanny I. Familial Alzheimer disease-linked mutations specifically disrupt Ca2+ leak function of presenilin 1. J Clin Invest. 2007;117(5):1230-9.

9. Reddy K, Cusack CL, Nnah IC, Khayati K, Saqcena C, Huynh TB, et al. Dysregulation of Nutrient Sensing and CLEARance in Presenilin Deficiency. Cell Rep. 2016;14(9):2166-79.

10. Tu H, Nelson O, Bezprozvanny A, Wang Z, Lee SF, Hao YH, et al. Presenilins form ER Ca2+ leak channels, a function disrupted by familial Alzheimer's disease-linked mutations. Cell. 2006;126(5):981-93.

11. Orr ME, Oddo S. Autophagic/lysosomal dysfunction in Alzheimer's disease. Alzheimers Res Ther. 2013;5(5):53.

12. Supnet $C$, Bezprozvanny I. Presenilins function in ER calcium leak and Alzheimer's disease pathogenesis. Cell Calcium. 2011;50(3):303-9.

13. Zare-Shahabadi A, Masliah E, Johnson GV, Rezaei N. Autophagy in Alzheimer's disease. Rev Neurosci. 2015;26(4):385-95.

14. Daigle I, Li C. apl-1, a Caenorhabditis elegans gene encoding a protein related to the human betaamyloid protein precursor. Proc Natl Acad Sci U S A. 1993;90(24):12045-9. 
15. McColl G, Roberts BR, Pukala TL, Kenche VB, Roberts CM, Link CD, et al. Utility of an improved model of amyloid-beta (Abeta(1)(-)(4)(2)) toxicity in Caenorhabditis elegans for drug screening for Alzheimer's disease. Mol Neurodegener. 2012;7:57.

16. Ashkavand Z, Sarasija S, Ryan KC, Laboy JT, Norman KR. Corrupted ER-mitochondrial calcium homeostasis promotes the collapse of proteostasis. Aging Cell. 2020;19(1):e13065.

17. Chan SL, Mayne M, Holden CP, Geiger JD, Mattson MP. Presenilin-1 mutations increase levels of ryanodine receptors and calcium release in PC12 cells and cortical neurons. J Biol Chem. 2000;275(24):18195-200.

18. Oksanen M, Petersen AJ, Naumenko N, Puttonen $K$, Lehtonen S, Gubert Olive $M$, et al. PSEN1 Mutant iPSC-Derived Model Reveals Severe Astrocyte Pathology in Alzheimer's Disease. Stem Cell Reports. 2017;9(6):1885-97.

19. Sarasija S, Norman KR. Role of Presenilin in Mitochondrial Oxidative Stress and Neurodegeneration in Caenorhabditis elegans. Antioxidants (Basel). 2018;7(9).

20. Stutzmann GE, Caccamo A, LaFerla FM, Parker I. Dysregulated IP3 signaling in cortical neurons of knock-in mice expressing an Alzheimer's-linked mutation in presenilin1 results in exaggerated $\mathrm{Ca} 2+$ signals and altered membrane excitability. J Neurosci. 2004;24(2):508-13.

21. Weidling IW, Swerdlow RH. Mitochondria in Alzheimer's disease and their potential role in Alzheimer's proteostasis. Exp Neurol. 2020;330:113321.

22. Nixon RA. Amyloid precursor protein and endosomal-lysosomal dysfunction in Alzheimer's disease: inseparable partners in a multifactorial disease. FASEB J. 2017;31(7):2729-43.

23. Sarasija S, Laboy JT, Ashkavand Z, Bonner J, Tang Y, Norman KR. Presenilin mutations deregulate mitochondrial $\mathrm{Ca}(2+)$ homeostasis and metabolic activity causing neurodegeneration in Caenorhabditis elegans. Elife. 2018;7.

24. Lapierre LR, De Magalhaes Filho CD, McQuary PR, Chu CC, Visvikis O, Chang JT, et al. The TFEB orthologue HLH-30 regulates autophagy and modulates longevity in Caenorhabditis elegans. Nat Commun. 2013;4:2267.

25. Timmons L, Fire A. Specific interference by ingested dsRNA. Nature. 1998;395(6705):854.

26. Kamath RS, Fraser AG, Dong Y, Poulin G, Durbin R, Gotta M, et al. Systematic functional analysis of the Caenorhabditis elegans genome using RNAi. Nature. 2003;421(6920):231-7.

27. Hartl FU, Bracher A, Hayer-Hartl M. Molecular chaperones in protein folding and proteostasis. Nature. 2011;475(7356):324-32.

28. Morawe T, Hiebel C, Kern A, Behl C. Protein homeostasis, aging and Alzheimer's disease. Mol Neurobiol. 2012;46(1):41-54.

29. Balch WE, Morimoto RI, Dillin A, Kelly JW. Adapting proteostasis for disease intervention. Science. 2008;319(5865):916-9.

30. Chiti F, Dobson CM. Protein Misfolding, Amyloid Formation, and Human Disease: A Summary of Progress Over the Last Decade. Annu Rev Biochem. 2017;86:27-68. 
31. Hipp MS, Park SH, Hartl FU. Proteostasis impairment in protein-misfolding and -aggregation diseases. Trends Cell Biol. 2014;24(9):506-14.

32. Klaips CL, Jayaraj GG, Hartl FU. Pathways of cellular proteostasis in aging and disease. J Cell Biol. 2018;217(1):51-63.

33. Kundra R, Ciryam P, Morimoto RI, Dobson CM, Vendruscolo M. Protein homeostasis of a metastable subproteome associated with Alzheimer's disease. Proc Natl Acad Sci U S A. 2017;114(28):E5703E11.

34. Lehrbach NJ, Ruvkun G. Endoplasmic reticulum-associated SKN-1A/Nrf1 mediates a cytoplasmic unfolded protein response and promotes longevity. Elife. 2019;8.

35. Jia K, Levine B. Autophagy is required for dietary restriction-mediated life span extension in C. elegans. Autophagy. 2007;3(6):597-9.

36. Kang C, You YJ, Avery L. Dual roles of autophagy in the survival of Caenorhabditis elegans during starvation. Genes Dev. 2007;21(17):2161-71.

37. Melendez A, Talloczy Z, Seaman M, Eskelinen EL, Hall DH, Levine B. Autophagy genes are essential for dauer development and life-span extension in C. elegans. Science. 2003;301(5638):1387-91.

38. Xu S, Chisholm AD. C. elegans epidermal wounding induces a mitochondrial ROS burst that promotes wound repair. Dev Cell. 2014;31(1):48-60.

39. Springhorn A, Hoppe T. Western blot analysis of the autophagosomal membrane protein LGG-1/LC3 in Caenorhabditis elegans. Methods Enzymol. 2019;619:319-36.

40. Robida-Stubbs S, Glover-Cutter K, Lamming DW, Mizunuma M, Narasimhan SD, Neumann-Haefelin E, et al. TOR signaling and rapamycin influence longevity by regulating SKN-1/Nrf and DAF-16/FoxO. Cell Metab. 2012;15(5):713-24.

41. Chong CM, Ke M, Tan Y, Huang Z, Zhang K, Ai N, et al. Presenilin 1 deficiency suppresses autophagy in human neural stem cells through reducing gamma-secretase-independent ERK/CREB signaling. Cell Death Dis. 2018;9(9):879.

42. Fedeli C, Filadi R, Rossi A, Mammucari C, Pizzo P. PSEN2 (presenilin 2) mutants linked to familial Alzheimer disease impair autophagy by altering $\mathrm{Ca}(2+)$ homeostasis. Autophagy. 2019;15(12):204462.

43. Kim J, Guan KL. mTOR as a central hub of nutrient signalling and cell growth. Nat Cell Biol. 2019;21(1):63-71.

44. Liu GY, Sabatini DM. mTOR at the nexus of nutrition, growth, ageing and disease. Nat Rev Mol Cell Biol. 2020;21(4):183-203.

45. Moullan N, Mouchiroud L, Wang X, Ryu D, Williams EG, Mottis A, et al. Tetracyclines Disturb Mitochondrial Function across Eukaryotic Models: A Call for Caution in Biomedical Research. Cell Rep. 2015;10(10):1681-91.

46. Pan CL, Peng CY, Chen $\mathrm{CH}$, McIntire S. Genetic analysis of age-dependent defects of the Caenorhabditis elegans touch receptor neurons. Proc Natl Acad Sci U S A. 2011;108(22):9274-9. 
47. Tank EM, Rodgers KE, Kenyon C. Spontaneous age-related neurite branching in Caenorhabditis elegans. J Neurosci. 2011;31(25):9279-88.

48. Toth ML, Melentijevic I, Shah L, Bhatia A, Lu K, Talwar A, et al. Neurite sprouting and synapse deterioration in the aging Caenorhabditis elegans nervous system. J Neurosci. 2012;32(26):8778-90.

49. Mair W, Morantte I, Rodrigues AP, Manning G, Montminy M, Shaw RJ, et al. Lifespan extension induced by AMPK and calcineurin is mediated by CRTC-1 and CREB. Nature. 2011;470(7334):404-8.

50. Zhang Y, Lanjuin A, Chowdhury SR, Mistry M, Silva-Garcia CG, Weir HJ, et al. Neuronal TORC1 modulates longevity via AMPK and cell nonautonomous regulation of mitochondrial dynamics in $\mathrm{C}$. elegans. Elife. 2019;8.

51. Su KH, Dai CK. mTORC1 senses stresses: Coupling stress to proteostasis. Bioessays. 2017;39(5).

52. Morley JF, Brignull HR, Weyers JJ, Morimoto RI. The threshold for polyglutamine-expansion protein aggregation and cellular toxicity is dynamic and influenced by aging in Caenorhabditis elegans. $P$ Natl Acad Sci USA. 2002;99(16):10417-22.

53. Zevian SC, Yanowitz JL. Methodological considerations for heat shock of the nematode Caenorhabditis elegans. Methods. 2014;68(3):450-7.

54. Sarasija S, Norman KR. A gamma-Secretase Independent Role for Presenilin in Calcium Homeostasis Impacts Mitochondrial Function and Morphology in Caenorhabditis elegans. Genetics. 2015;201(4):1453-+.

55. Fatouros C, Pir GJ, Biernat J, Koushika SP, Mandelkow E, Mandelkow EM, et al. Inhibition of tau aggregation in a novel Caenorhabditis elegans model of tauopathy mitigates proteotoxicity. Hum Mol Genet. 2012;21(16):3587-603.

56. Sure GR, Chatterjee A, Mishra N, Sabharwal V, Devireddy S, Awasthi A, et al. UNC-16/JIP3 and UNC$76 / F E Z 1$ limit the density of mitochondria in C. elegans neurons by maintaining the balance of anterograde and retrograde mitochondrial transport. Sci Rep-Uk. 2018;8.

57. Hansen M, Chandra A, Mitic LL, Onken B, Driscoll M, Kenyon C. A role for autophagy in the extension of lifespan by dietary restriction in C-elegans. Plos Genet. 2008;4(2).

58. De Strooper B. Aph-1, Pen-2, and nicastrin with presenilin generate an active gamma-secretase complex. Neuron. 2003;38(1):9-12.

59. Kimberly WT, LaVoie MJ, Ostaszewski BL, Ye WJ, Wolfe MS, Selkoe DJ. gamma-secretase is a membrane protein complex comprised of presenilin, nicastrin, aph-1, and pen-2. P Natl Acad Sci USA. 2003;100(11):6382-7.

60. Gertsik N, Chiu D, Li YM. Complex regulation of gamma-secretase: from obligatory to modulatory subunits. Front Aging Neurosci. 2015;6.

61. Area-Gomez E, Castillo MDL, Tambini MD, Guardia-Laguarta C, de Groof AJC, Madra M, et al. Upregulated function of mitochondria-associated ER membranes in Alzheimer disease. Embo J. 2012;31(21):4106-23. 
62. Area-Gomez E, de Groof AJC, Boldogh I, Bird TD, Gibson GE, Koehler CM, et al. Presenilins Are Enriched in Endoplasmic Reticulum Membranes Associated with Mitochondria. Am J Pathol. 2009;175(5):1810-6.

63. Bhaskar K, Miller M, Chludzinski A, Herrup K, Zagorski M, Lamb BT. The PI3K-Akt-mTOR pathway regulates A beta oligomer induced neuronal cell cycle events. Molecular Neurodegeneration. 2009;4.

64. Caccamo A, Maldonado MA, Majumder S, Medina DX, Holbein W, Magri A, et al. Naturally Secreted Amyloid-beta Increases Mammalian Target of Rapamycin (mTOR) Activity via a PRAS40-mediated Mechanism. Journal of Biological Chemistry. 2011;286(11):8924-32.

65. Li X, Alafuzoff I, Soininen H, Winblad B, Pei JJ. Levels of mTOR and its downstream targets 4E-BP1, eEF2, and eEF2 kinase in relationships with tau in Alzheimer's disease brain. Febs J. 2005;272(16):4211-20.

66. Tramutola A, Lanzillotta C, Arena A, Barone E, Perluigi M, Di Domenico F. Increased Mammalian Target of Rapamycin Signaling Contributes to the Accumulation of Protein Oxidative Damage in a Mouse Model of Down's Syndrome. Neurodegener Dis. 2016;16(1-2):62-8.

67. Ma T, Hoeffer CA, Capetillo-Zarate E, Yu FM, Wong H, Lin MT, et al. Dysregulation of the mTOR Pathway Mediates Impairment of Synaptic Plasticity in a Mouse Model of Alzheimer's Disease. Plos One. 2010;5(9).

68. An WL, Cowburn RF, Li L, Braak H, Alafuzoff I, Iqbal K, et al. Up-regulation of phosphorylated/activated p70 S6 kinase and its relationship to neurofibrillary pathology in Alzheimer's disease (vol 163, pg 591, 2003). Am J Pathol. 2003;163(6):2645-.

69. Cai Z, Zhao B, Li KS, Zhang LQ, Li CH, Quazi SH, et al. Mammalian target of rapamycin: A valid therapeutic target through the autophagy pathway for alzheimer's disease? J Neurosci Res. 2012;90(6):1105-18.

70. Caccamo A, Magri A, Medina DX, Wisely EV, Lopez-Aranda MF, Silva AJ, et al. mTOR regulates tau phosphorylation and degradation: implications for Alzheimer's disease and other tauopathies. Aging Cell. 2013;12(3):370-80.

71. Khurana V, Lu YR, Steinhilb ML, Oldham S, Shulman JM, Feany MB. TOR-mediated cell-cycle activation causes neurodegeneration in a Drosophila tauopathy model. Curr Biol. 2006;16(3):230-41.

72. Finkel T, Menazza S, Holmstrom KM, Parks RJ, Liu J, Sun J, et al. The ins and outs of mitochondrial calcium. Circ Res. 2015;116(11):1810-9.

73. Starkov AA, Chinopoulos C, Fiskum G. Mitochondrial calcium and oxidative stress as mediators of ischemic brain injury. Cell Calcium. 2004;36(3-4):257-64.

74. Williams GS, Boyman L, Chikando AC, Khairallah RJ, Lederer WJ. Mitochondrial calcium uptake. Proc Natl Acad Sci U S A. 2013;110(26):10479-86.

75. Ham SJ, Lee D, Yoo H, Jun K, Shin H, Chung J. Decision between mitophagy and apoptosis by Parkin via VDAC1 ubiquitination. Proc Natl Acad Sci U S A. 2020;117(8):4281-91.

76. Jadiya P, Kolmetzky DW, Tomar D, Di Meco A, Lombardi AA, Lambert JP, et al. Impaired mitochondrial calcium efflux contributes to disease progression in models of Alzheimer's disease. Nat Commun. 
2019;10(1):3885.

77. Ludtmann MHR, Kostic M, Horne A, Gandhi S, Sekler I, Abramov AY. LRRK2 deficiency induced mitochondrial $\mathrm{Ca}(2+)$ efflux inhibition can be rescued by $\mathrm{Na}(+) / \mathrm{Ca}(2+) / \mathrm{Li}(+)$ exchanger upregulation. Cell Death Dis. 2019;10(4):265.

78. Muller M, Ahumada-Castro U, Sanhueza M, Gonzalez-Billault C, Court FA, Cardenas C. Mitochondria and Calcium Regulation as Basis of Neurodegeneration Associated With Aging. Front Neurosci. 2018;12:470.

79. Verma M, Callio J, Otero PA, Sekler I, Wills ZP, Chu CT. Mitochondrial Calcium Dysregulation Contributes to Dendrite Degeneration Mediated by PD/LBD-Associated LRRK2 Mutants. J Neurosci. 2017;37(46):11151-65.

80. Lee KS, Huh S, Lee S, Wu Z, Kim AK, Kang HY, et al. Altered ER-mitochondria contact impacts mitochondria calcium homeostasis and contributes to neurodegeneration in vivo in disease models. Proc Natl Acad Sci U S A. 2018;115(38):E8844-E53.

81. Soman SK, Bazala M, Keatinge M, Bandmann O, Kuznicki J. Restriction of mitochondrial calcium overload by mcu inactivation renders a neuroprotective effect in zebrafish models of Parkinson's disease. Biol Open. 2019;8(10).

82. Calvo-Rodriguez M, Hou SS, Snyder AC, Kharitonova EK, Russ AN, Das S, et al. Increased mitochondrial calcium levels associated with neuronal death in a mouse model of Alzheimer's disease. Nat Commun. 2020;11(1):2146.

83. Caccamo A, De Pinto V, Messina A, Branca C, Oddo S. Genetic reduction of mammalian target of rapamycin ameliorates Alzheimer's disease-like cognitive and pathological deficits by restoring hippocampal gene expression signature. J Neurosci. 2014;34(23):7988-98.

84. Crino PB. The mTOR signalling cascade: paving new roads to cure neurological disease. Nat Rev Neurol. 2016;12(7):379-92.

85. Kaur A, Sharma S. Mammalian target of rapamycin (mTOR) as a potential therapeutic target in various diseases. Inflammopharmacology. 2017;25(3):293-312.

86. Lin AL, Zheng W, Halloran JJ, Burbank RR, Hussong SA, Hart MJ, et al. Chronic rapamycin restores brain vascular integrity and function through NO synthase activation and improves memory in symptomatic mice modeling Alzheimer's disease. J Cereb Blood Flow Metab. 2013;33(9):1412-21.

87. Ozcelik S, Fraser G, Castets P, Schaeffer V, Skachokova Z, Breu K, et al. Rapamycin attenuates the progression of tau pathology in P301S tau transgenic mice. Plos One. 2013;8(5):e62459.

88. Siman R, Cocca R, Dong Y. The mTOR Inhibitor Rapamycin Mitigates Perforant Pathway Neurodegeneration and Synapse Loss in a Mouse Model of Early-Stage Alzheimer-Type Tauopathy. Plos One. 2015;10(11):e0142340.

89. Spilman P, Podlutskaya N, Hart MJ, Debnath J, Gorostiza O, Bredesen D, et al. Inhibition of mTOR by rapamycin abolishes cognitive deficits and reduces amyloid-beta levels in a mouse model of Alzheimer's disease. Plos One. 2010;5(4):e9979. 
Figures

A

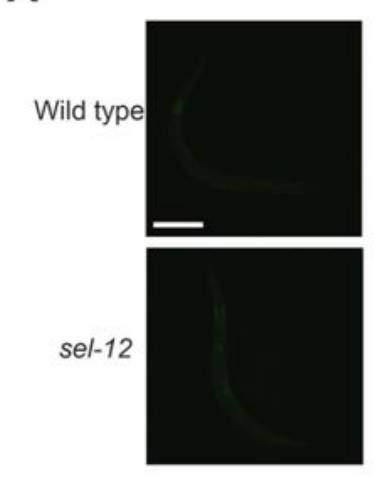

C

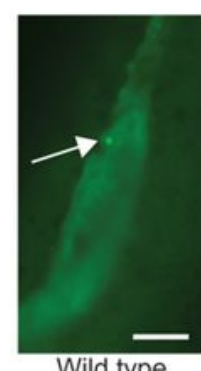

$\mathrm{E}$

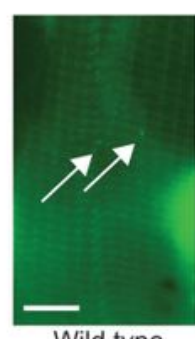

Wild type
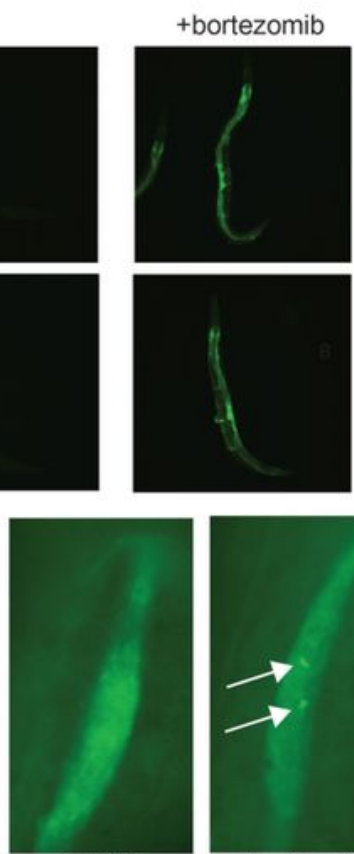

sel-12

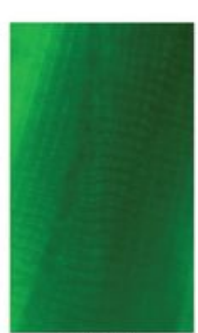

sel-12

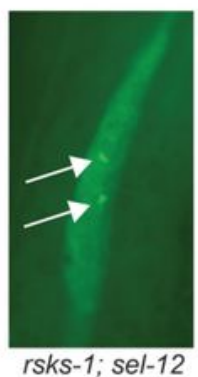

D
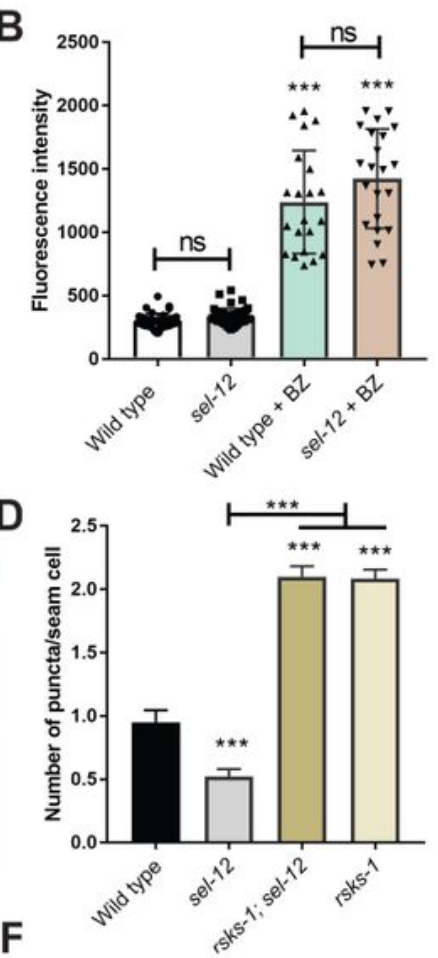

$\mathrm{F}$

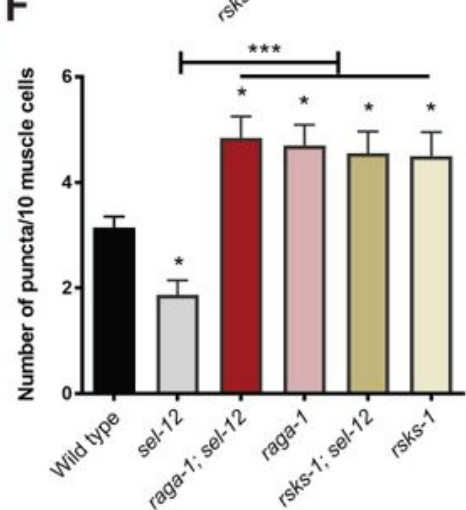

G
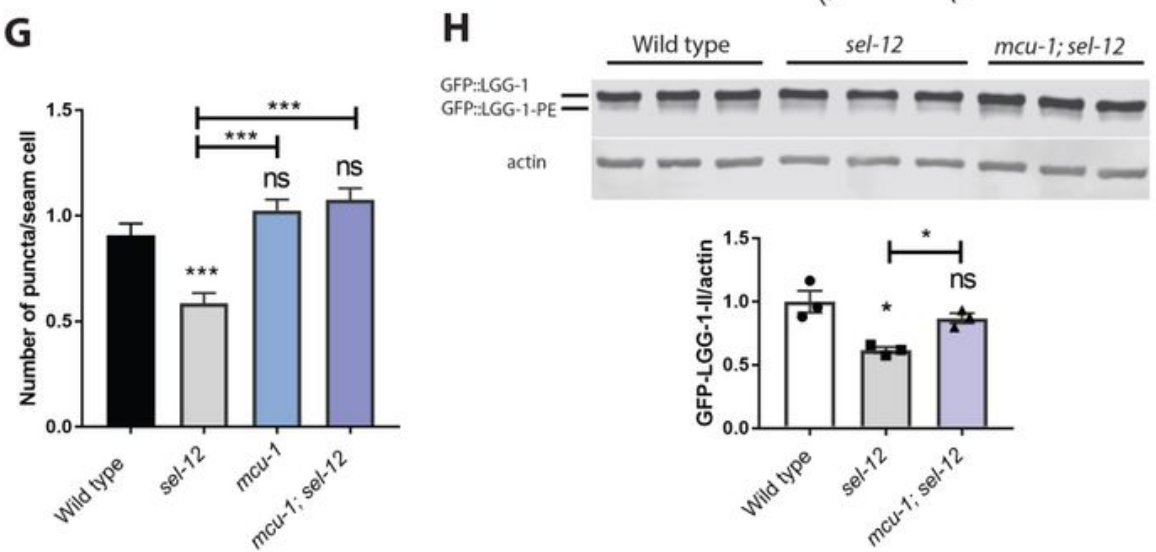

\section{Figure 1}

sel-12 mutants show reduced autophagy that is restored by mTORC1 inhibition. (A and B) Representative images of wild type and sel-12(ty11) animals expressing rpl-28p::ub(G76V)::GFP (mgls77) treated with proteasome inhibitor $(10 \mu \mathrm{M}$ bortezomib) overnight (scale bar $=100 \mu \mathrm{m})(\mathrm{A})$ and quantification of GFP 
intensity (B). $\mathrm{n}$ is $\geq 25$ animals. ( $\mathrm{C}$ and $\mathrm{E}$ ) Representative images of hypodermal seam cells (scale bar = $10 \mu \mathrm{m})(\mathrm{C})$ or muscle (scale bar $=10 \mu \mathrm{m})(\mathrm{E})$ expressing GFP::LGG-1/LC3 puncta (arrows) as a reporter for autophagosome formation. (D and F) Quantification of GFP:LGG-1/LC3 puncta in seam cells (D) ( $n=$ 125 seam cells) or muscle (F) ( $n=90$ muscle cells). (G) Quantification of GFP:LGG-1/LC3 puncta in seam cells ( $n=200$ seam cells). ( $\mathrm{H}$ and $\mathrm{I})$. Western blot to determine the levels of PE-conjugated (lipidated) GFP::LGG-1 using an anti-GFP antibody, and (I) quantification of GFP::LGG-1-PE/actin. ns $P>0.05$, ${ }^{*} \mathrm{P}<0.05,{ }^{*} * \mathrm{P}<0.001$ using two-way ANOVA. Bars indicate mean $+/$ - SEM.

A

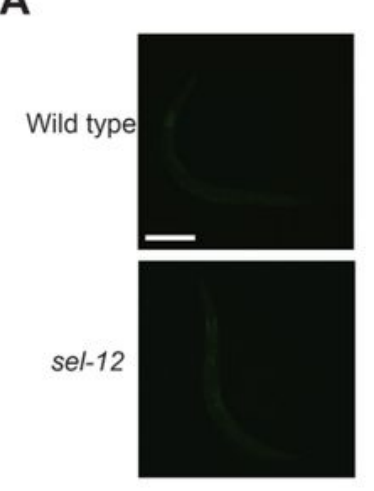

C

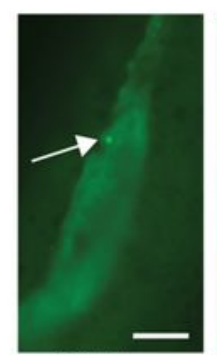

Wild type

E

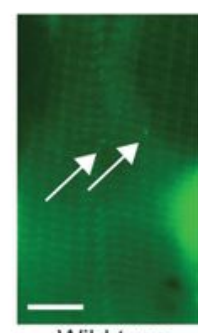

Wild type
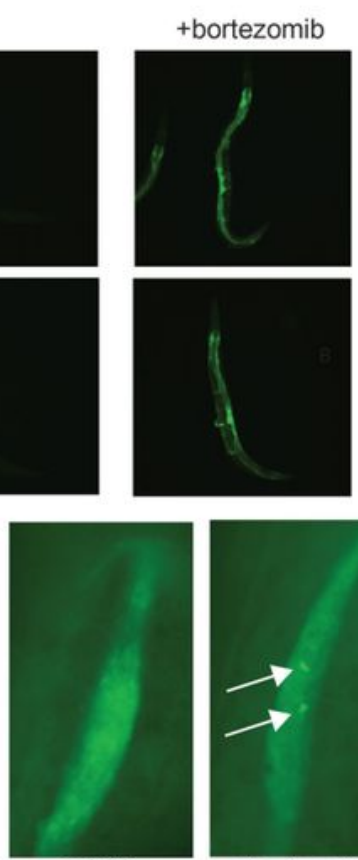

sel-12

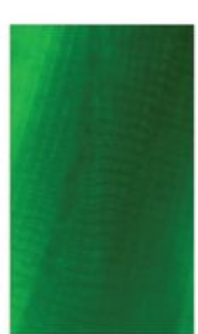

sel-12
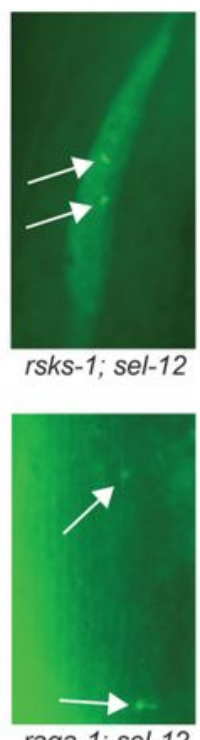

B

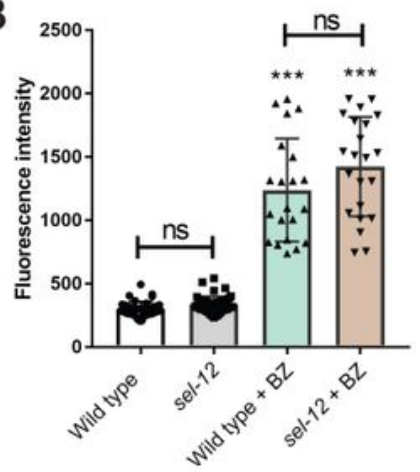

D
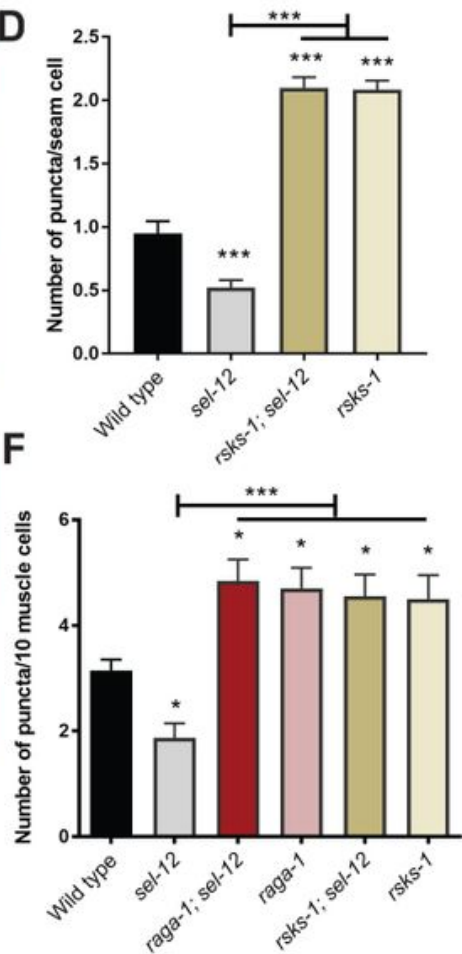

G

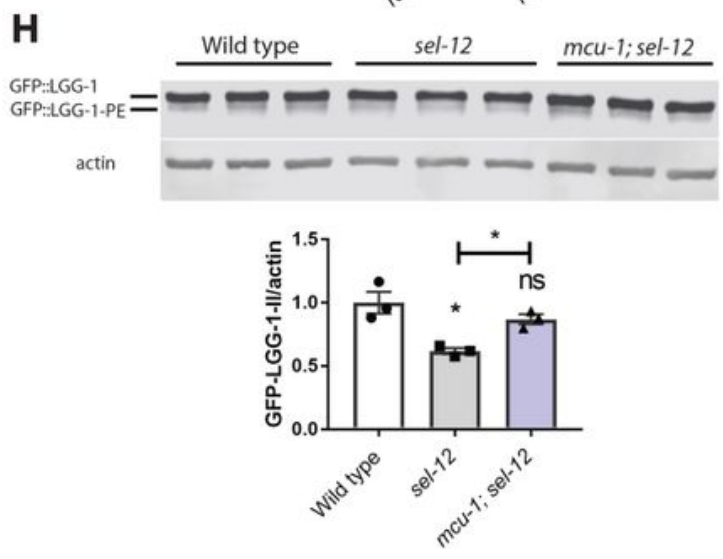




\section{Figure 1}

sel-12 mutants show reduced autophagy that is restored by mTORC1 inhibition. (A and B) Representative images of wild type and sel-12(ty11) animals expressing rpl-28p::ub(G76V)::GFP (mgls77) treated with proteasome inhibitor $(10 \mu \mathrm{M}$ bortezomib) overnight (scale bar $=100 \mu \mathrm{m})(\mathrm{A})$ and quantification of GFP intensity (B). $n$ is $\geq 25$ animals. ( $C$ and $E$ ) Representative images of hypodermal seam cells (scale bar = $10 \mu \mathrm{m})(\mathrm{C})$ or muscle (scale bar $=10 \mu \mathrm{m})(\mathrm{E})$ expressing GFP::LGG-1/LC3 puncta (arrows) as a reporter for autophagosome formation. (D and F) Quantification of GFP:LGG-1/LC3 puncta in seam cells (D) ( $n=$ 125 seam cells) or muscle ( $F)(n=90$ muscle cells). (G) Quantification of GFP:LGG-1/LC3 puncta in seam cells $(n=200$ seam cells). ( $\mathrm{H}$ and $\mathrm{I})$. Western blot to determine the levels of PE-conjugated (lipidated) GFP::LGG-1 using an anti-GFP antibody, and (I) quantification of GFP::LGG-1-PE/actin. ns $P>0.05$, ${ }^{*} \mathrm{P}<0.05,{ }^{* *} \mathrm{P}<0.001$ using two-way ANOVA. Bars indicate mean $+/$ - SEM. 
A
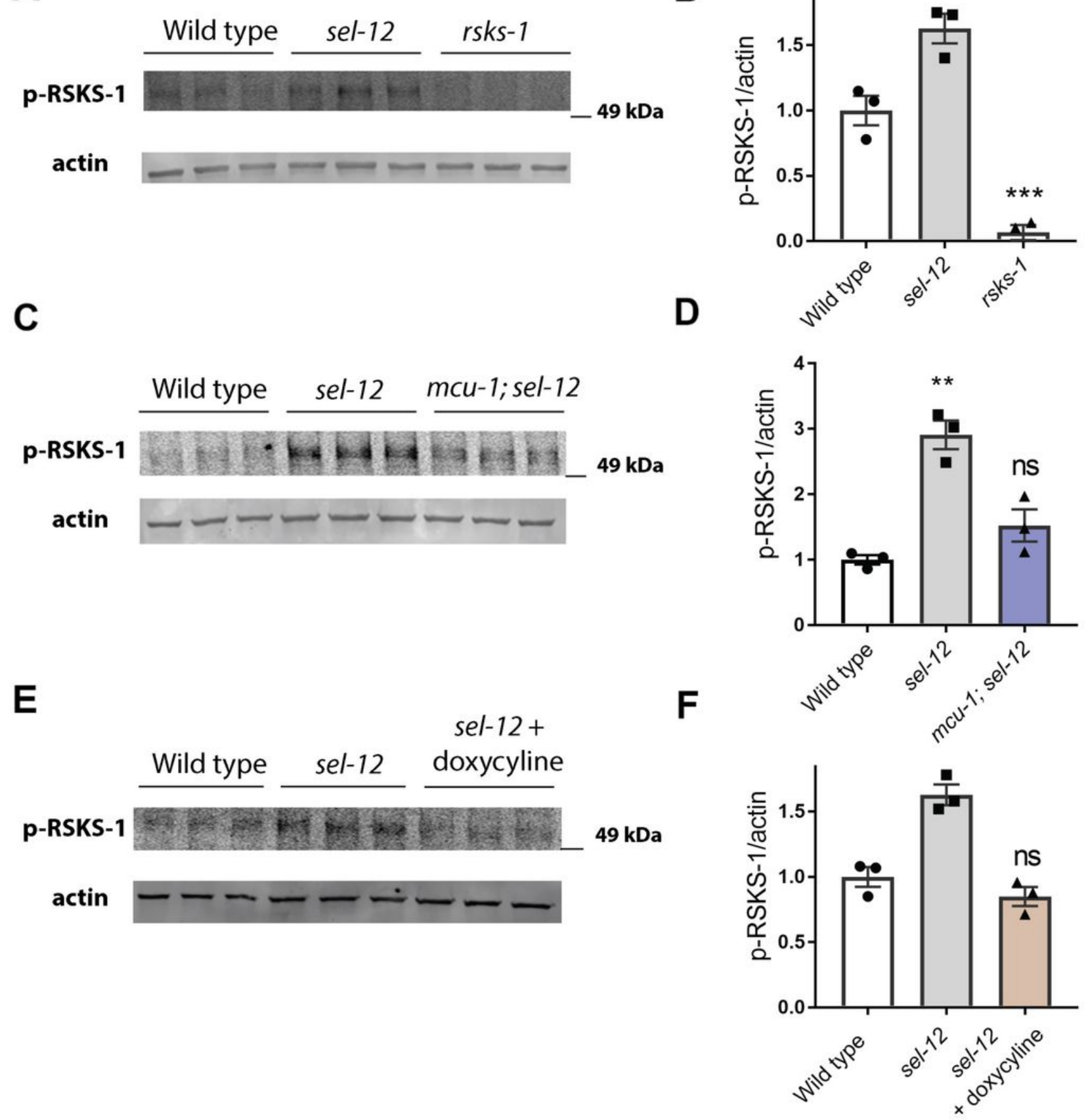

Figure 2

Reduction of mitochondrial calcium uptake or activity prevents mTORC1 hyperactivation in sel-12 mutants. (A, C, and E) Western blot of p-RSKS-1 and actin (loading control), and (B, D, and F) quantification of $\mathrm{p}-\mathrm{RSKS}-1 /$ actin. Error bars indicate SEM. ns $\mathrm{P}>0.05,{ }^{\star *} \mathrm{P}<0.01$ using ANOVA with Tukey Test. $\mathrm{n} \geq 3$. 
A
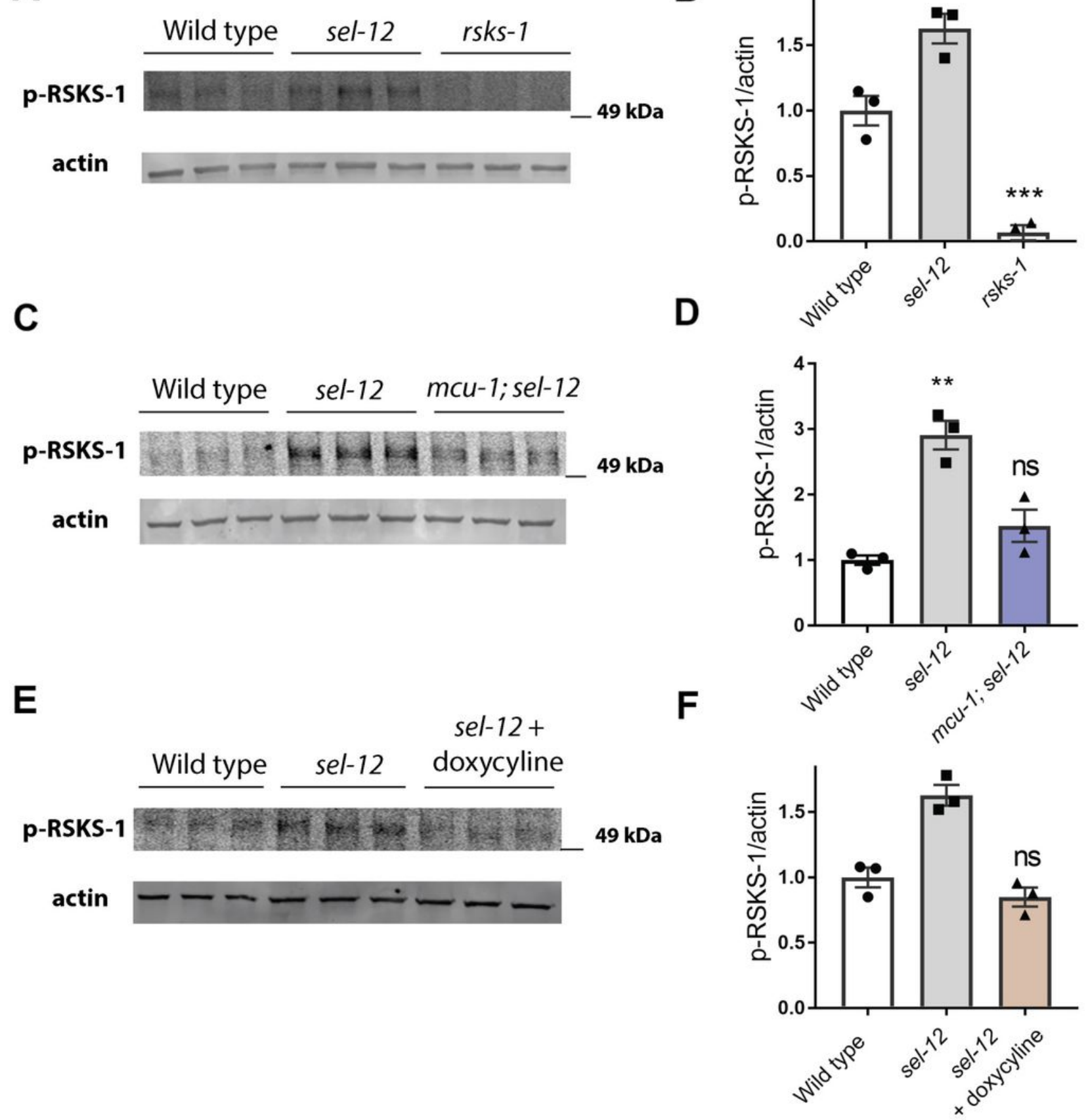

Figure 2

Reduction of mitochondrial calcium uptake or activity prevents mTORC1 hyperactivation in sel-12 mutants. (A, C, and E) Western blot of p-RSKS-1 and actin (loading control), and (B, D, and F) quantification of $p$-RSKS-1/actin. Error bars indicate SEM. ns $P>0.05,{ }^{* *} P<0.01$ using ANOVA with Tukey Test. $\mathrm{n} \geq 3$. 
A
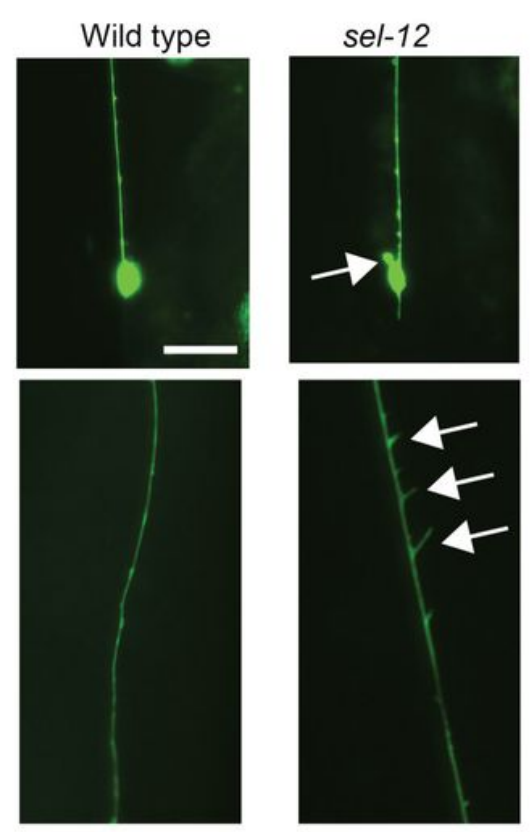

$\mathbf{F}$

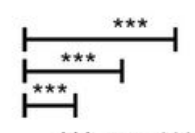

is $n$ ns $n$

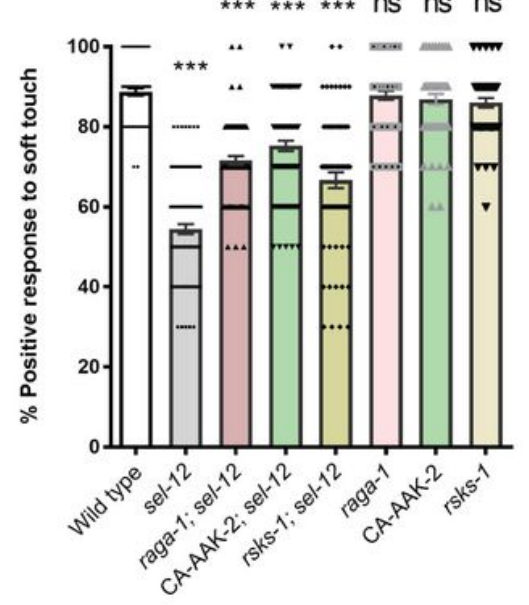

B

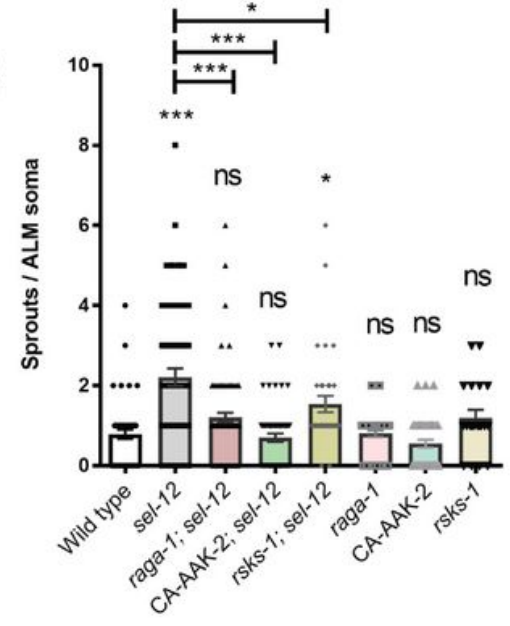

D

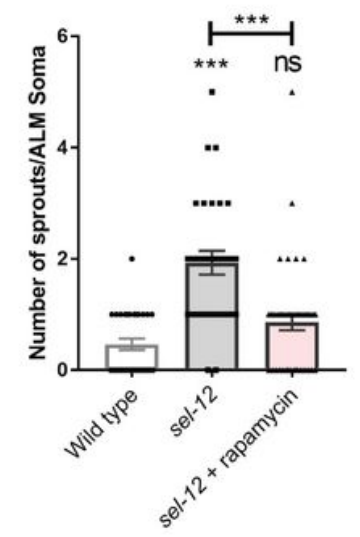

G

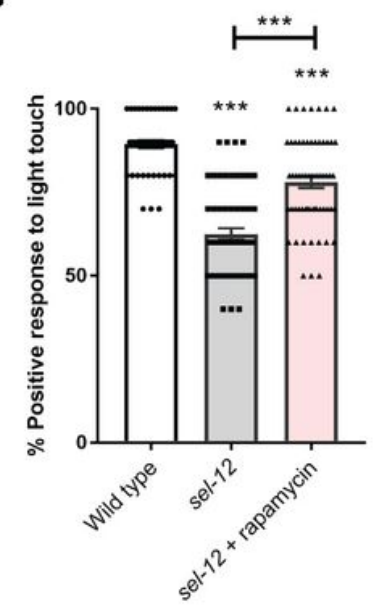

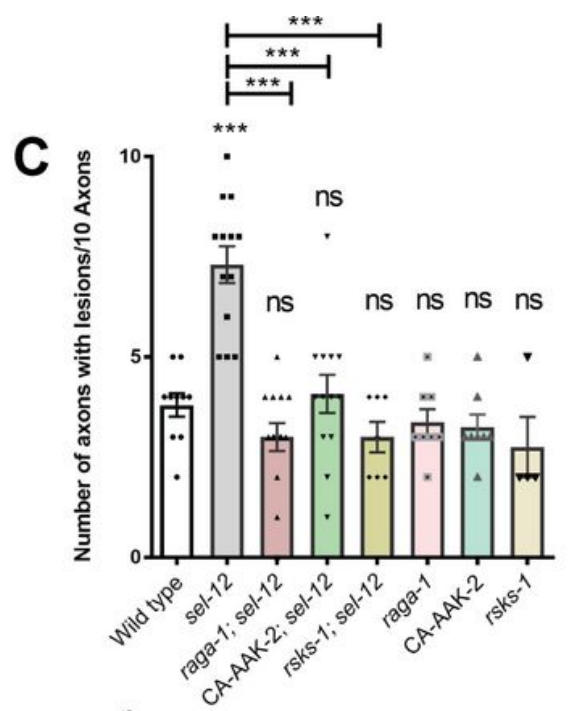

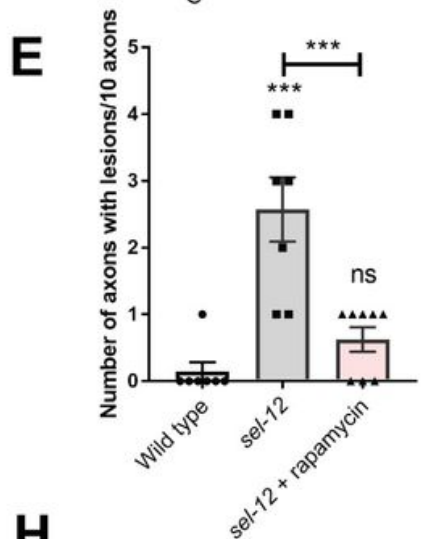

H

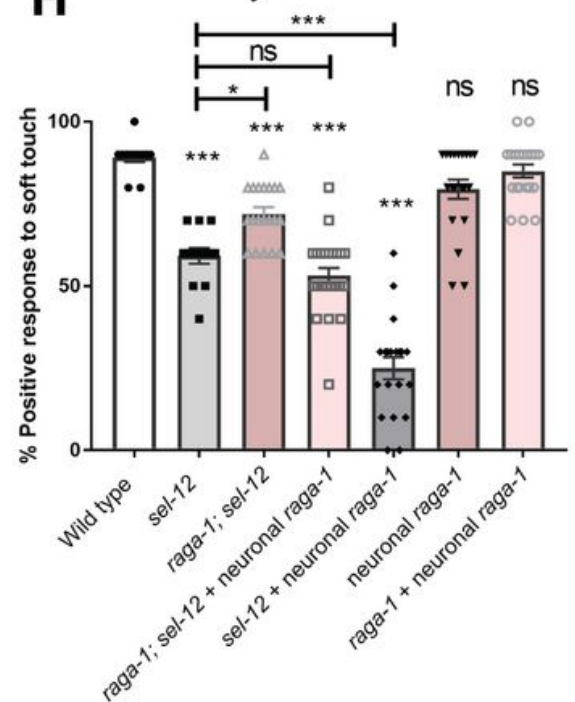

Figure 3

Inhibition of mTORC1 restores the structural integrity of mechanosensory neurons and improves mechanosensation in sel-12 mutants. (A) Representative images of wild type ALM mechanosensory neuron, sel-12 ALM with aberrant sprouts, wild type PLM axon, and sel-12 PLM axon with aberrant lesions (scale bar $=10 \mu \mathrm{m})$. (B and $C$ ) Average number of aberrant ectopic sprouts per ALM soma $(n \geq 30$ animals) (B) and number of aberrant lesions per 10 ALM/PLM axons ( $n \geq 60$ axons) (C) using the bzls166 [mec-4p:::mCherry] reporter. (D and E) Number of aberrant sprouts (D) and axonal lesions $(E)$ in 
animals grown in presence or absence of rapamycin using the zdls5 [mec-4p::GFP] reporter. ( $F$ and $G$ ) Soft touch assay quantifying percentage response to anterior and posterior soft touch. ( $\mathrm{n} \geq 50$ animals.). $(\mathrm{H})$ Soft touch assay in animals expressing raga- $1 \mathrm{cDNA}$ in neurons driven by the rab-3 promoter. $(\mathrm{n}=20$ animals). Error bars indicate SEM. ns $P>0.05,{ }^{*} P<0.05$, *** $P<0.001$ using ANOVA with Tukey Test.

A
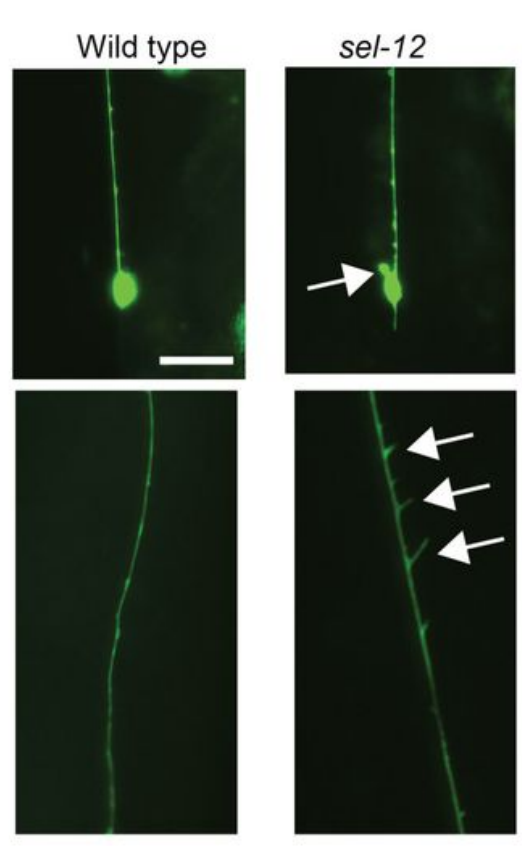

$\mathbf{F}$
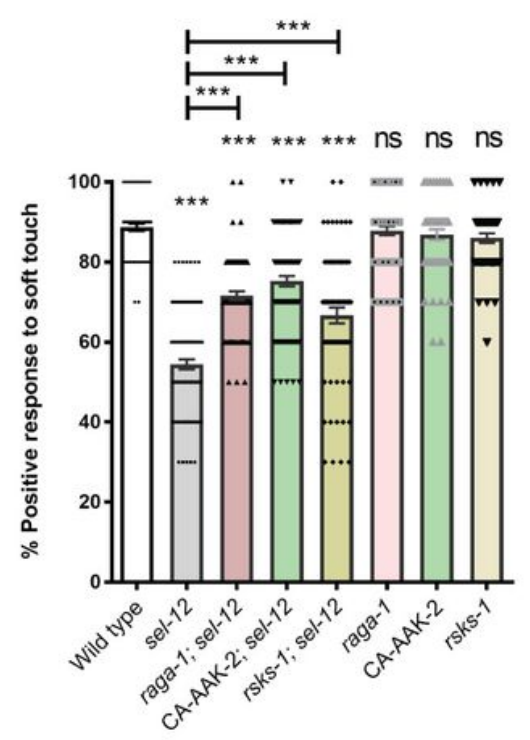
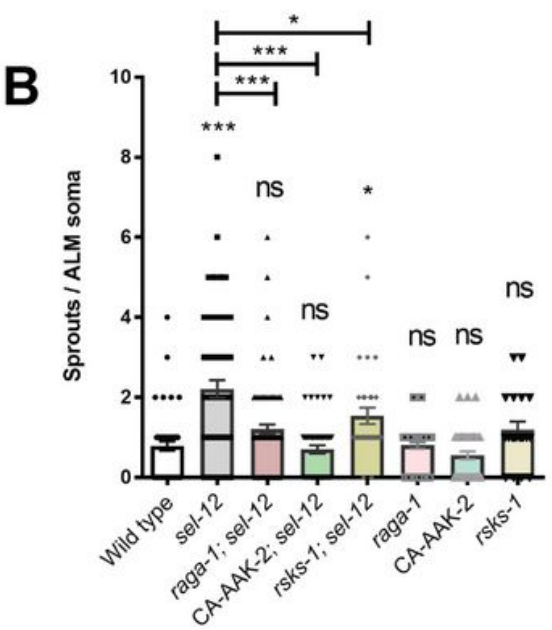

D

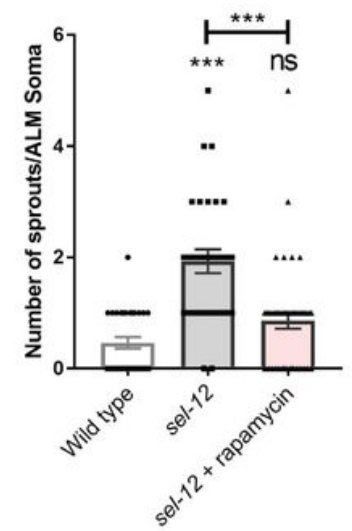

G

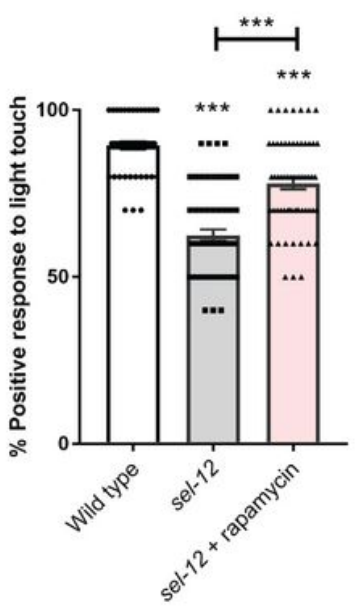

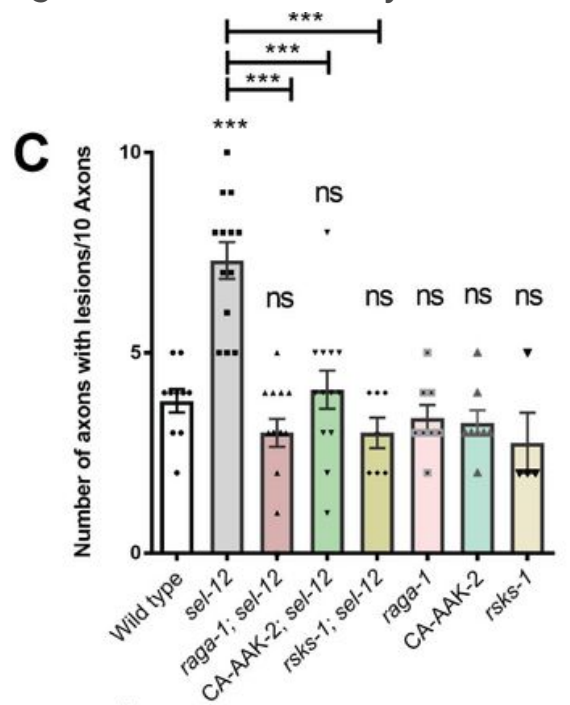

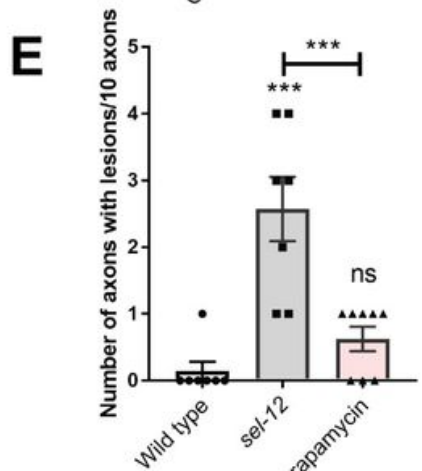

H

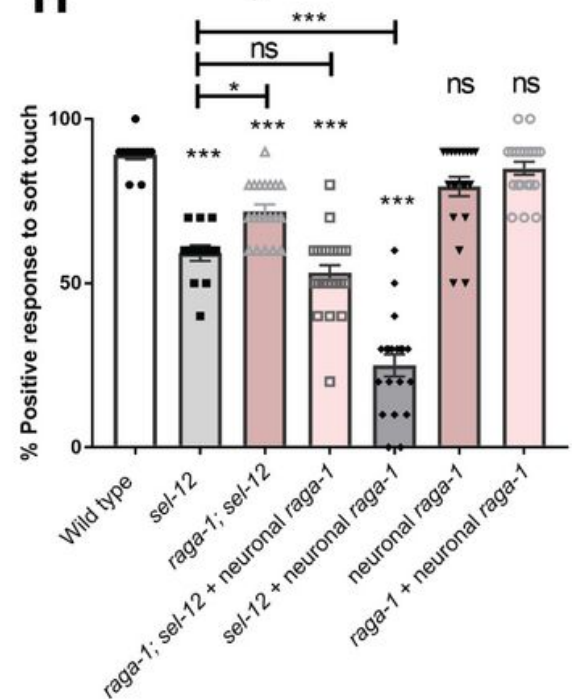

Figure 3

Inhibition of mTORC1 restores the structural integrity of mechanosensory neurons and improves mechanosensation in sel-12 mutants. (A) Representative images of wild type ALM mechanosensory 
neuron, sel-12 ALM with aberrant sprouts, wild type PLM axon, and sel-12 PLM axon with aberrant lesions (scale bar $=10 \mu \mathrm{m})$. (B and C) Average number of aberrant ectopic sprouts per ALM soma $(\mathrm{n} \geq 30$ animals) (B) and number of aberrant lesions per 10 ALM/PLM axons ( $n \geq 60$ axons) (C) using the bzls166 [mec-4p::mCherry] reporter. (D and E) Number of aberrant sprouts (D) and axonal lesions (E) in animals grown in presence or absence of rapamycin using the zdls5 [mec-4p::GFP] reporter. ( $F$ and $G$ ) Soft touch assay quantifying percentage response to anterior and posterior soft touch. ( $\mathrm{n} \geq 50$ animals.). $(\mathrm{H})$ Soft touch assay in animals expressing raga-1 cDNA in neurons driven by the rab-3 promoter. $(\mathrm{n}=20$ animals). Error bars indicate SEM. ns $P>0.05,{ }^{*} \mathrm{P}<0.05$, ${ }^{\star * *} \mathrm{P}<0.001$ using ANOVA with Tukey Test.

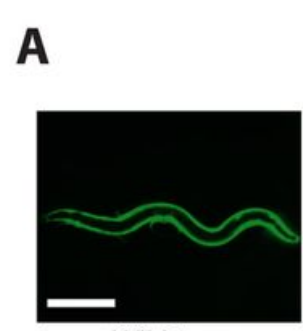

Wild type

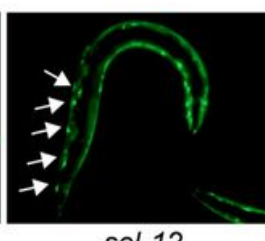

sel-12

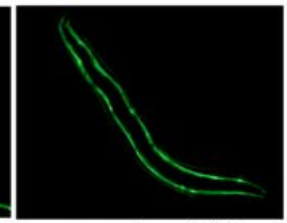

raga-1; sel-12

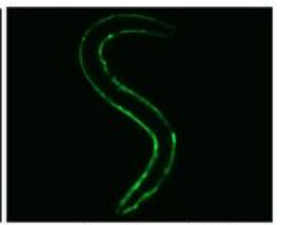

rsks-1; sel-12

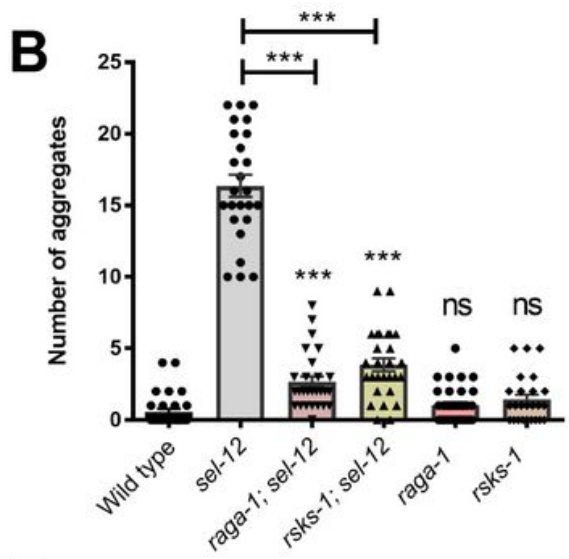

C

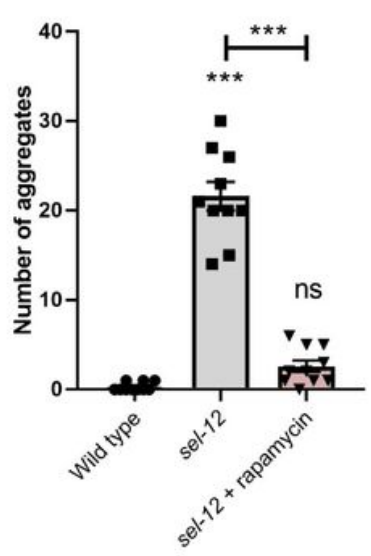

D

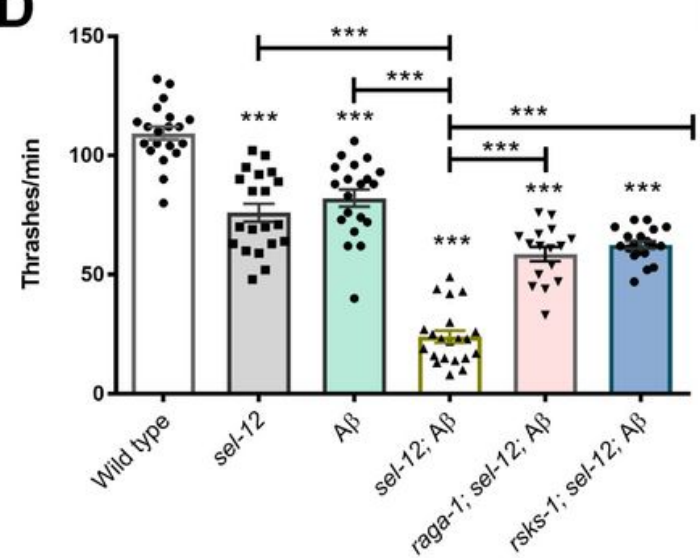

E
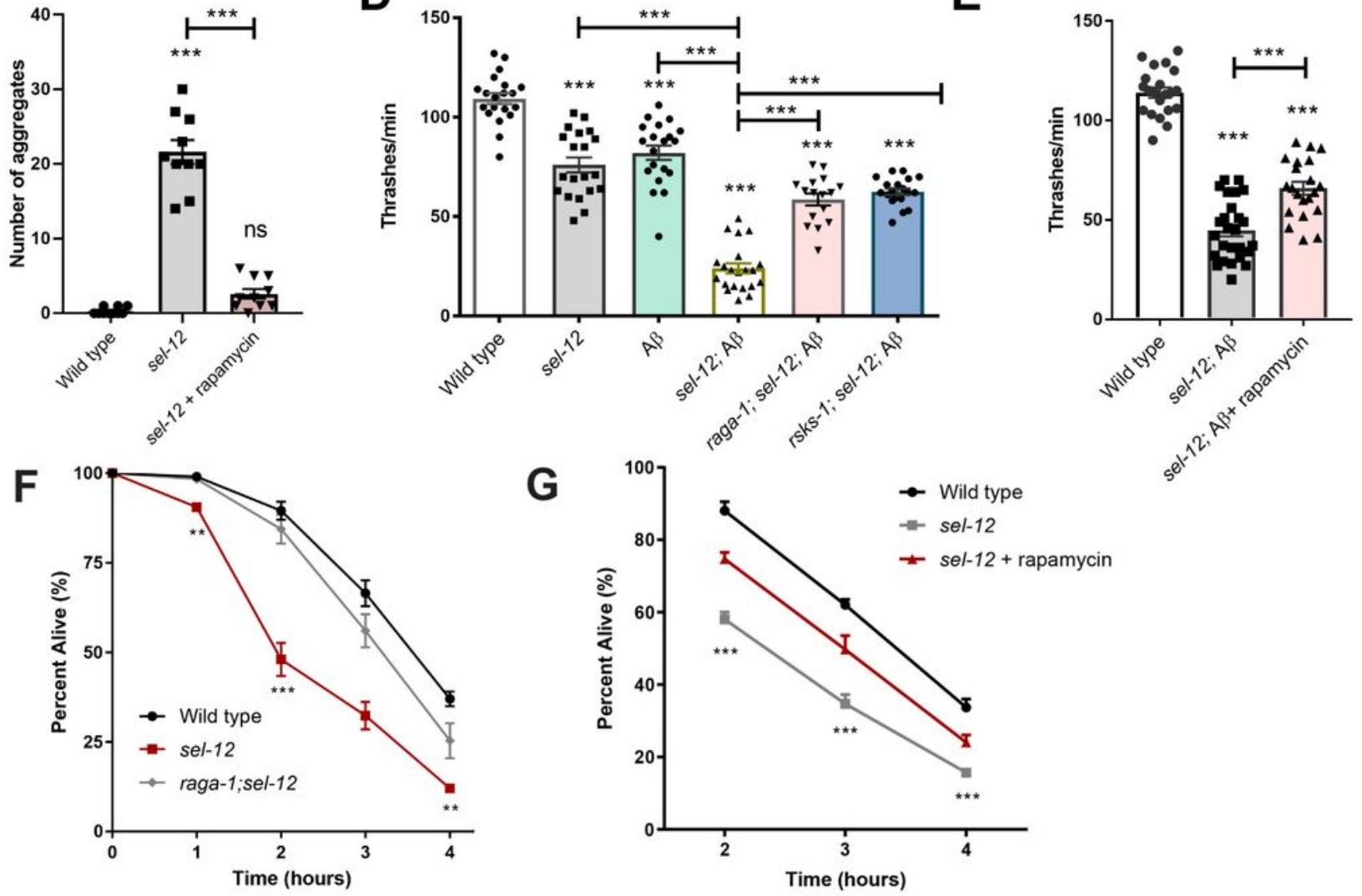

Figure 4

Inhibition of mTORC1 rescues loss of proteostasis in sel-12 mutants. (A) Representative images of animals expressing polyQ35::YFP (scale bar $=100 \mu \mathrm{m})$. (B and $\mathrm{C}$ ) Mean number of polyQ aggregates 
observed in day 3 wild type, sel-12, raga-1; sel-12, rsks-1; sel-12 adults (B) or day 3 sel-12 adults following rapamycin treatment $(C)(n=30)$. (D, E) Mean thrashing rate in wild type, sel-12, raga-1; sel-12, or rsks-1; sel-12 mutants expressing human 1-42 A (dvls100) (D) or in 1-42 A -expressing sel-12(ty11) animals following rapamycin treatment $(E)(n=20$.) ( $F$ and $G)$ Sensitivity to heat stress. Animals were scored for survival after exposure to heat stress at $37^{\circ} \mathrm{C}$. (50 animals per strain, repeated twice). Error bars indicate SEM. ns $P>0.05,{ }^{*} P<0.05,{ }^{*} P<0.01, * \star \star P<0.001$ using one-way ANOVA $(A-E)$ and two-way ANOVA $(F, G)$ with Tukey Test.

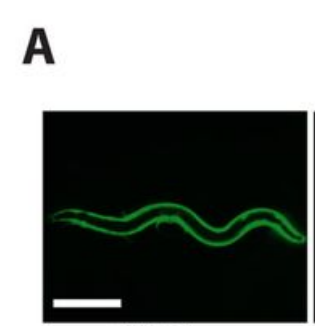

Wild type

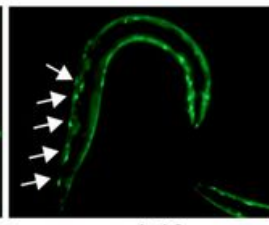

sel-12

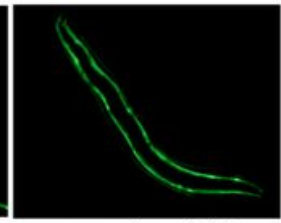

raga-1; sel-12

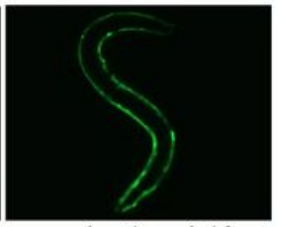

rsks-1; sel-12
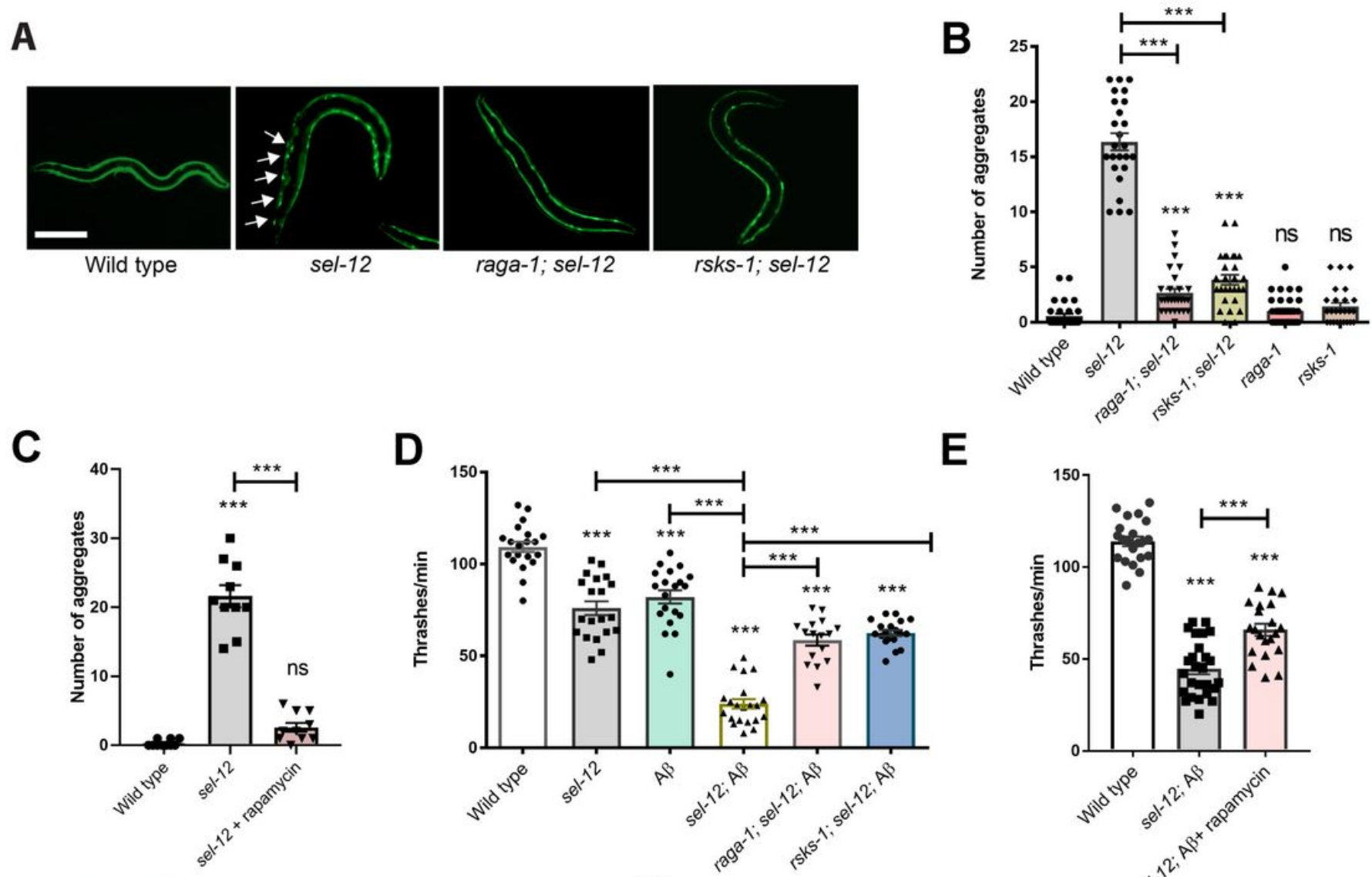

$\mathbf{E}$
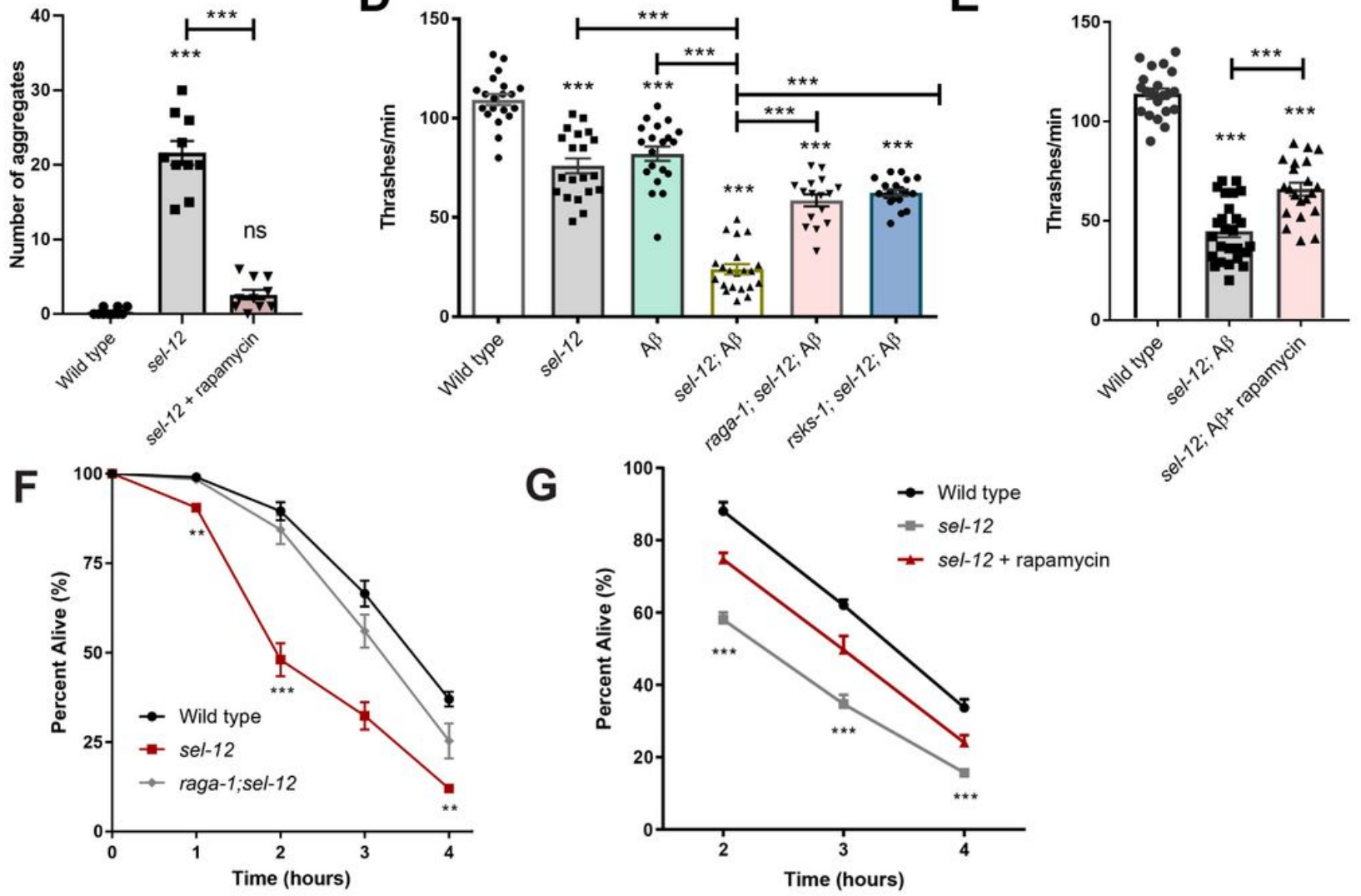

Figure 4

Inhibition of mTORC1 rescues loss of proteostasis in sel-12 mutants. (A) Representative images of animals expressing polyQ35::YFP (scale bar $=100 \mu \mathrm{m}$ ). (B and $\mathrm{C}$ ) Mean number of polyQ aggregates observed in day 3 wild type, sel-12, raga-1; sel-12, rsks-1; sel-12 adults (B) or day 3 sel-12 adults following 
rapamycin treatment $(C)(n=30)$. (D, E) Mean thrashing rate in wild type, sel-12, raga-1; sel-12, or rsks-1; sel-12 mutants expressing human 1-42 A (dvls100) (D) or in 1-42 A -expressing sel-12(ty11) animals following rapamycin treatment $(E)(n=20$.) $(F$ and $G)$ Sensitivity to heat stress. Animals were scored for survival after exposure to heat stress at $37^{\circ} \mathrm{C}$. (50 animals per strain, repeated twice). Error bars indicate SEM. ns $P>0.05,{ }^{*} P<0.05,{ }^{*} \mathrm{P}<0.01,{ }^{*} * \mathrm{P}<0.001$ using one-way ANOVA $(A-E)$ and two-way ANOVA $(F, G)$ with Tukey Test.

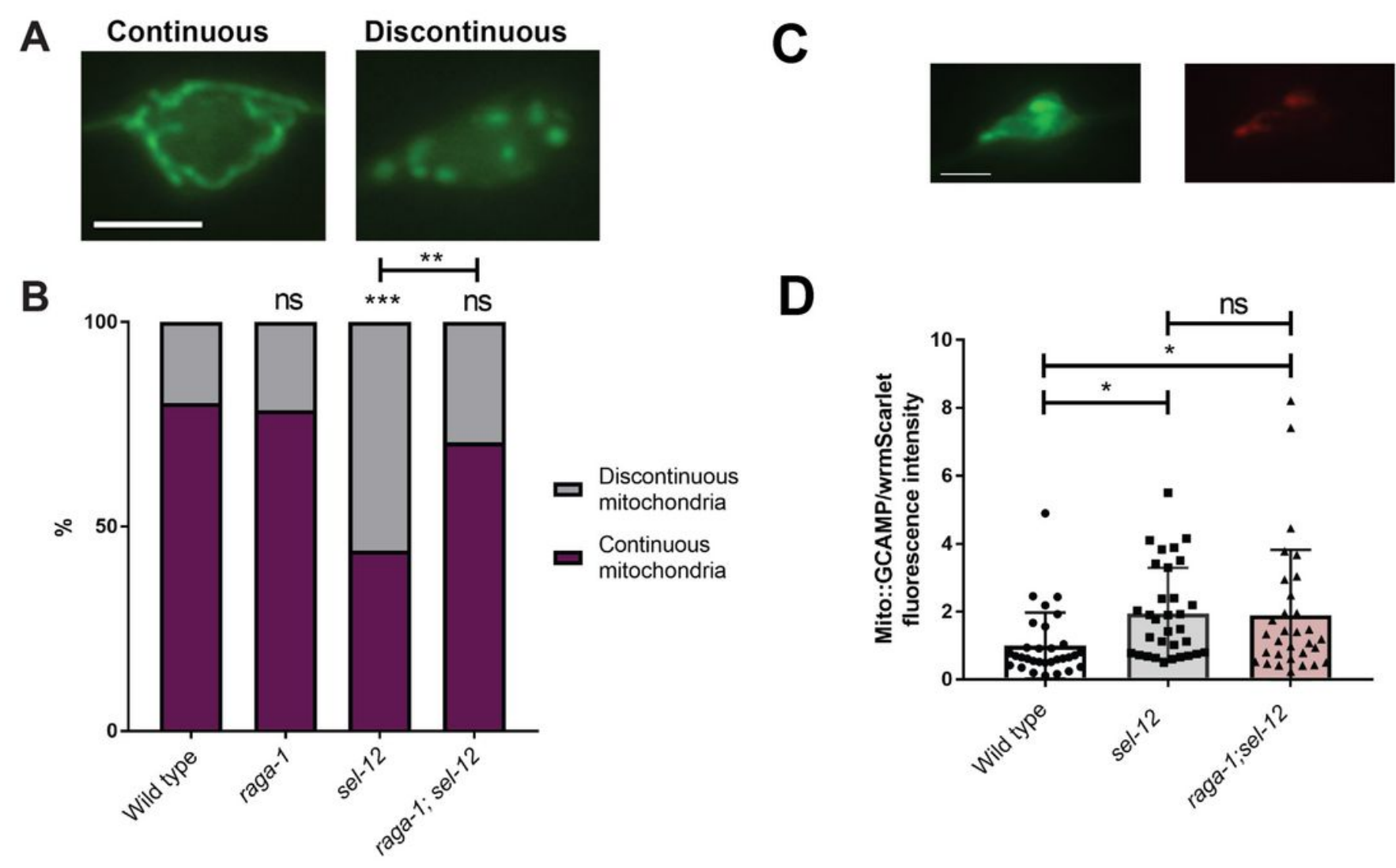

Figure 5

mTORC1 inhibition improves mitochondrial morphology but does not rescue elevated mitochondrial calcium in sel-12 mutants. (A) Representative images and quantification of ALM soma mitochondria in mechanosensory neurons using transgenic animals expressing mitochondrial localization signal tagged to GFP in the mechanosensory neurons (jsls609) (scale bar $=5 \mu \mathrm{m})$. $(n=50)$. (B) Quantification of mitochondrial morphology ( $n \geq 50$ animals). ( $C$ and $D)$ Representative images and quantification of mitochondrial matrix calcium levels using transgenic animals expressing mitochondrial-targeted GCaMP6 and wrmScarlet in the mechanosensory neurons to measure relative fluorescence intensity. ( $\mathrm{n}=$ 35). Error bars indicate $S E M$. ns $P>0.05,{ }^{*} P<0.05,{ }^{\star \star} P<0.01 * \star \star P<0.001$ using chi-squared test $(B)$ or ANOVA with Tukey Test (D). 

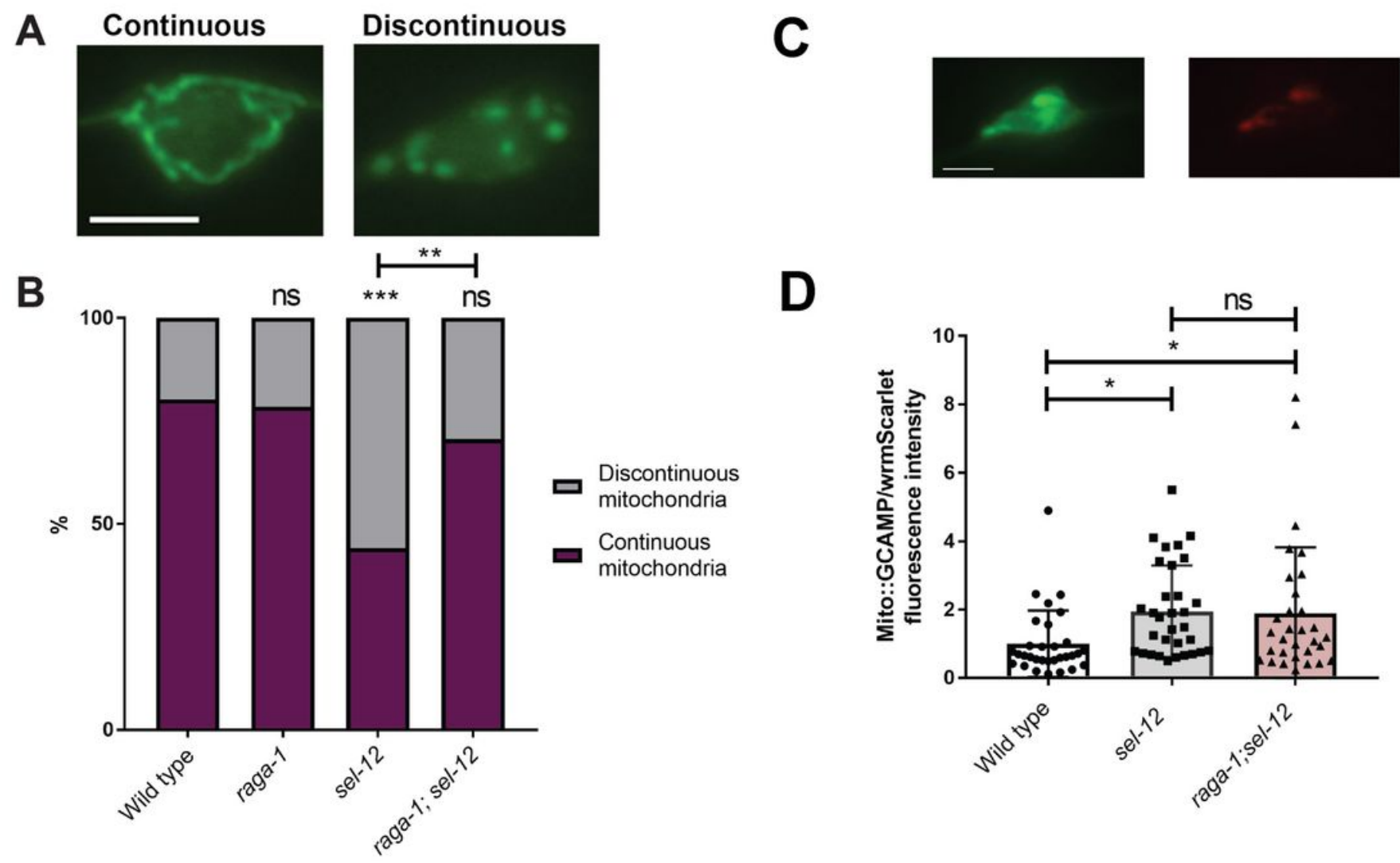

D

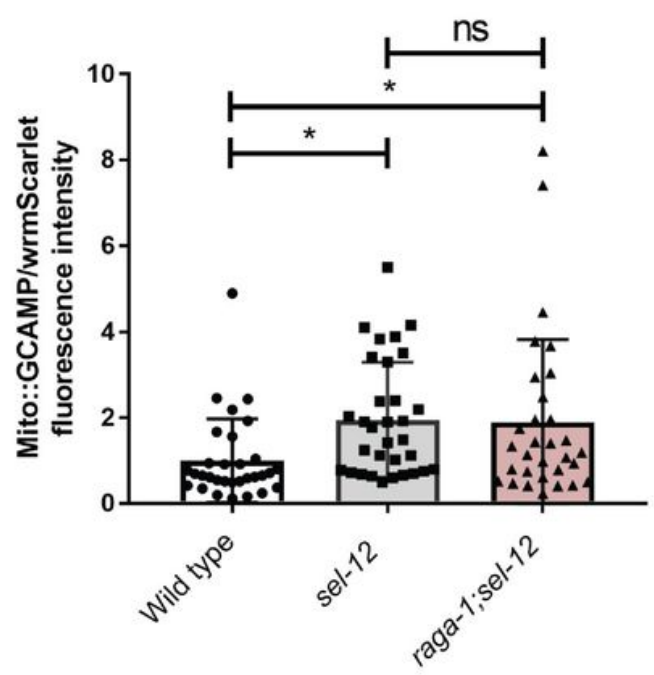

Figure 5

mTORC1 inhibition improves mitochondrial morphology but does not rescue elevated mitochondrial calcium in sel-12 mutants. (A) Representative images and quantification of ALM soma mitochondria in mechanosensory neurons using transgenic animals expressing mitochondrial localization signal tagged to GFP in the mechanosensory neurons (jsls609) (scale bar $=5 \mu \mathrm{m}) .(\mathrm{n}=50)$. (B) Quantification of mitochondrial morphology ( $n \geq 50$ animals). (C and D) Representative images and quantification of mitochondrial matrix calcium levels using transgenic animals expressing mitochondrial-targeted GCaMP6 and wrmScarlet in the mechanosensory neurons to measure relative fluorescence intensity. ( $n=$ 35). Error bars indicate $S E M$. ns $P>0.05,{ }^{*} P<0.05, \star \star P<0.01 * \star \star P<0.001$ using chi-squared test $(B)$ or ANOVA with Tukey Test (D). 


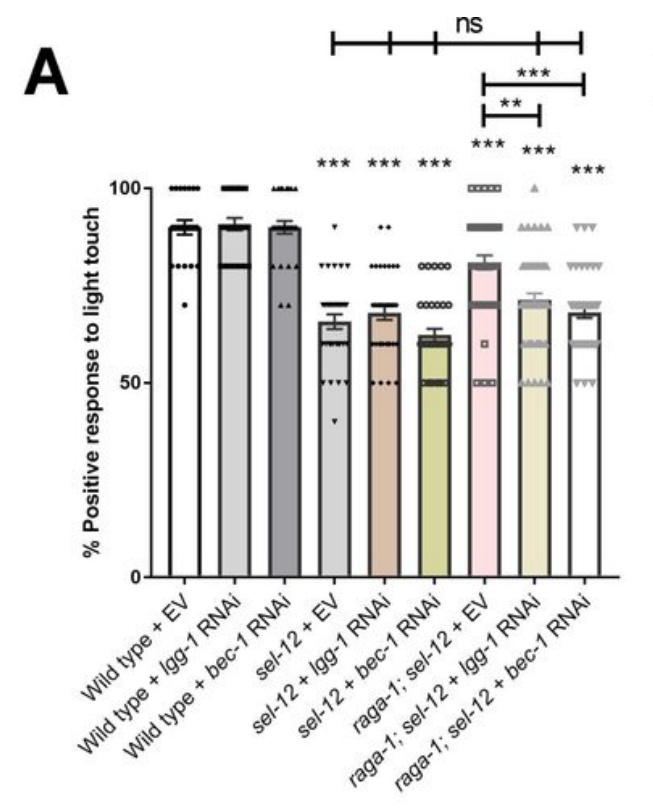

в
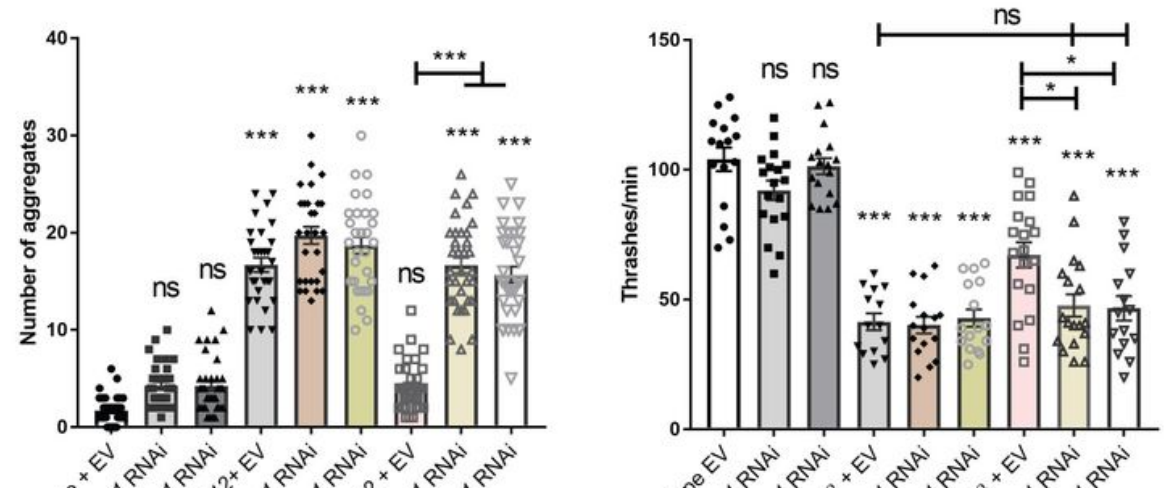

C
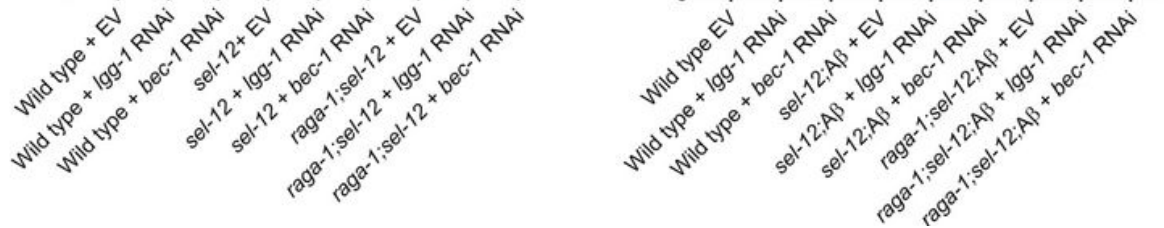

Figure 6

Rescue of proteostasis and behavior mediated by mTORC1 inhibition is dependent on autophagy. Percentage response to soft touch (A), number of thrashes per minute (B) or number of Q35 aggregates (C) in worms grown on EV, Igg-1, or bec-1 RNAi. ns $P>0.05$, ${ }^{*} P<0.05$, ${ }^{\star *} P<0.01$, ${ }^{\star *} * P<0.001$ using two-way ANOVA. Bars indicate mean and SEM of $\geq 20$ animals.
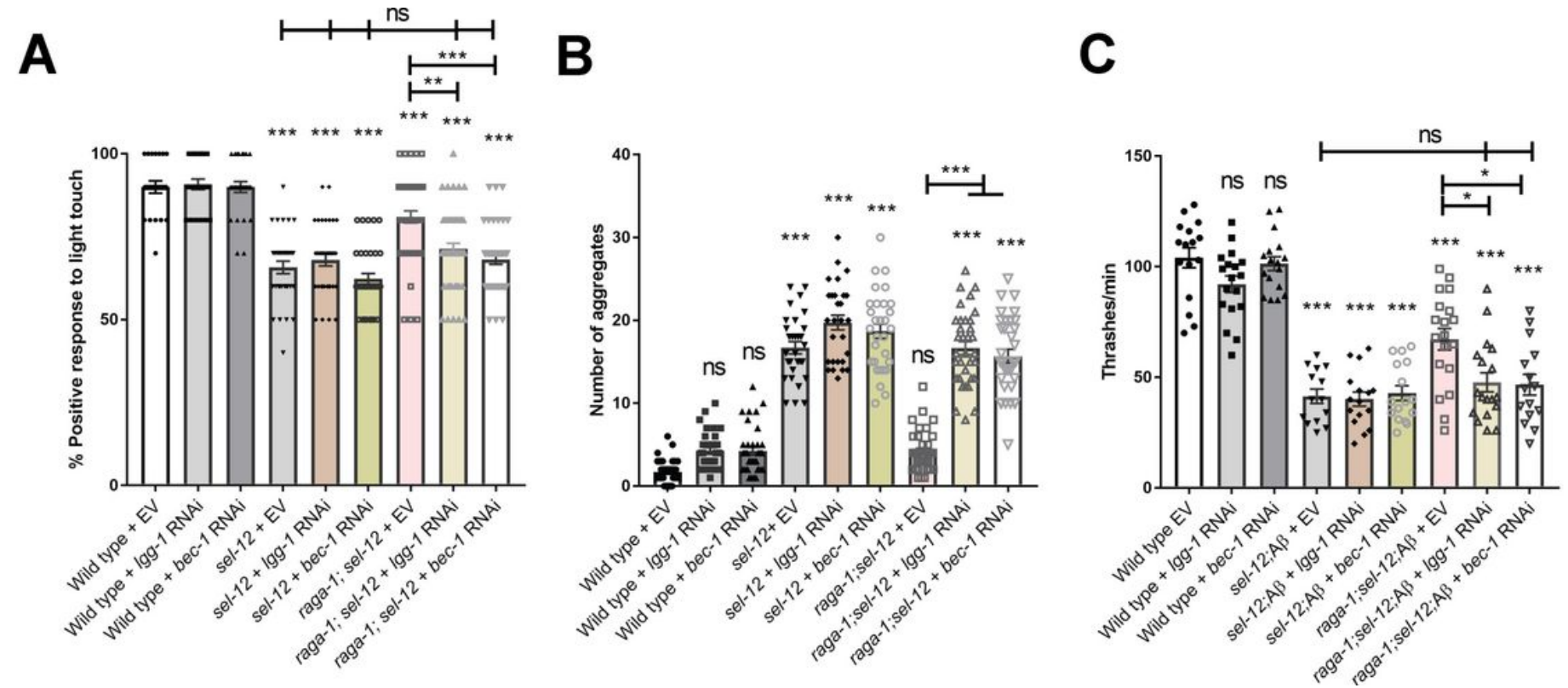

Figure 6

Rescue of proteostasis and behavior mediated by mTORC1 inhibition is dependent on autophagy. Percentage response to soft touch (A), number of thrashes per minute (B) or number of Q35 aggregates (C) in worms grown on EV, Igg-1, or bec-1 RNAi. ns $P>0.05$, ${ }^{*} P<0.05$, ${ }^{\star *} P<0.01$, ${ }^{\star \star *} P<0.001$ using two-way ANOVA. Bars indicate mean and SEM of $\geq 20$ animals. 


\section{Supplementary Files}

This is a list of supplementary files associated with this preprint. Click to download.

- FigureS1.jpg

- FigureS1.jpg

- Figures2.jpg

- Figures2.jpg

- FigureS3.jpg

- FigureS3.jpg

- FigureS4.jpg

- FigureS4.jpg 